\title{
A POSTERIORI ERROR ESTIMATION FOR THE DUAL MIXED FINITE ELEMENT METHOD FOR THE $p$-LAPLACIAN IN A POLYGONAL DOMAIN
}

\author{
E. CREUSE \\ LAMAV, Université de Valenciennes, Le Mont Houy \\ 59313 Valenciennes Cedex 09, France \\ and \\ INRIA Futurs Lille, Equipe SIMPAF \\ USTL, Cité scientifique \\ 59655 Villeneuve d'Ascq Cedex, France \\ emmanuel.creuse@univ-valenciennes.fr \\ M. FARHLOUL * \\ Département de Mathématiques et de Statistique, Université de Moncton \\ Moncton, N.B., E1A 3E9 Canada \\ farhlom@umoncton.ca \\ L. PAQUET \\ LAMAV, Université de Valenciennes, Le Mont Houy \\ 59313 Valenciennes Cedex 09, France \\ luc.paquet@univ-valenciennes.fr
}

\begin{abstract}
For the discrete solution of the dual mixed formulation for the $p$-Laplace equation, we define two residues $R$ and $r$. Then we bound the norm of the errors on the two unknowns in terms of the norms of these two residues. Afterwards, we bound the norms of these two residues by functions of two error estimators whose expressions involve at the very most the datum and the computed quantities. We next explain how the discretized dual mixed formulation is hybridized and solved. We close our paper by numerical tests for $p=1.8$ and $p=3$ firstly to corroborate the orders of convergence established by M. Farhloul and H. Manouzi [1], and secondly to experimentally verify the reliability of our a posteriori error estimates.
\end{abstract}

Keywords: p-Laplacian, dual mixed FEM, a posteriori error estimators, Helmholtz decomposition

AMS Subject Classification: 65N15, 65N30, 65N50, 35J60

* Corresponding author 


\section{Introduction}

Let us fix a bounded plane domain $\Omega$ with a polygonal boundary. More precisely, we assume that $\Omega$ is a simply connected domain and that its boundary $\Gamma$ is the union of a finite number of linear segments $\bar{\Gamma}_{j}, 1 \leq j \leq n_{e}\left(\Gamma_{j}\right.$ is assumed to be an open segment). In this domain we consider the $p$-Laplace equation

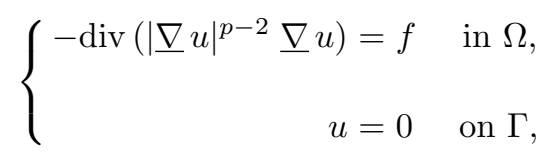

where $1<p<\infty, \underline{\nabla} u$ is the vector gradient of $u$,

$$
|\underline{\nabla} u|=\left(\sum_{i=1}^{2}\left(\frac{\partial u}{\partial x_{i}}\right)^{2}\right)^{\frac{1}{2}}
$$

and $f$ is a given function in $L^{q}(\Omega)$ ( $q$ conjugate of $p$ ). This equation occurs in many mathematical models of physical processes such as nonlinear diffusion and filtration, power-law materials, and viscoelastic materials.

In Farhloul and Manouzi [1], these authors have introduced a mixed finite element method for problem (0.1) based on the introduction of $\underline{\sigma}=|\underline{\nabla} u|^{p-2} \underline{\nabla} u$ as a new unknown and its approximation by the lowest degree Raviart-Thomas finite element (cf. Thomas [2], Raviart and Thomas [3]). The old unknown $u$ is approximated by a piecewise constant function on the triangulation of $\Omega$. They have proved a priori error estimates without any restriction on $p$. In this paper, we are concerned by the construction of reliable a posteriori error estimators for $\underline{\sigma}_{h}$ the approximation of $\underline{\sigma}$ and $u_{h}$ the approximation of $u$.

In section 1, we firstly introduce two residues $R$ and $r$ and we bound the norms of the errors $\left\|\underline{\sigma}-\underline{\sigma}_{h}\right\|_{0, q, \Omega}$ and $\left\|u-u_{h}\right\|_{0, p, \Omega}$ in terms of these two residues $\left(\|\cdot\|_{0, p, \Omega}\right.$ denotes the $L^{p}$-norm in $\Omega$ and $\left.\frac{1}{q}=1-\frac{1}{p}\right)$. In this part of the work, we distinguish two cases : $p \geq 2$ and $1<p<2$. Secondly, using a Helmholtz type decomposition for arbitrary vector field $\underline{\tau} \in\left[L^{q}(\Omega)\right]^{2}$, we establish a bound of the norm of $R$ in terms of a local error estimator $\eta_{1}=\left(\sum_{K \in \mathcal{T}_{h}} \eta_{1}(K)^{p}\right)^{\frac{1}{p}}$, where $\mathcal{T}_{h}$ denotes the triangulation of $\Omega$ and $K$ an arbitrary triangle of $\mathcal{T}_{h} \cdot \eta_{1}(K)$ is function of $\underline{A}\left(\underline{\sigma}_{h}\right)=$ $\left|\underline{\sigma}_{h}\right|^{q-2} \underline{\sigma}_{h}, \operatorname{curl} \underline{\mathrm{A}}\left(\underline{\sigma}_{\mathrm{h}}\right)$, the tangential jump of $\underline{A}\left(\underline{\sigma}_{h}\right)$ at each interface of two triangles $\left[\left[\underline{A}\left(\underline{\sigma}_{h}\right) \cdot \underline{t}\right]\right]$ and the diameter $h_{K}$ of the triangle $K$.

The norm of $r$ is easily bounded in terms of the local error estimator

$$
\eta_{2}=\left(\sum_{K \in \mathcal{T}_{h}} \eta_{2}(K)^{q}\right)^{\frac{1}{q}}
$$

where $\eta_{2}(K)=\left\|f-P_{h}^{0} f\right\|_{0, q, K} . P_{h}^{0} f$ is the function constant on each $K \in \mathcal{T}_{h}$ equal on $K$ to the mean of $f$ on $K$. 
In section 2 of our paper, we introduce the hybrid formulation of the discrete mixed formulation by expressing that the normal component of $\underline{\sigma}_{h}$ is continuous at each interface between triangles of $\mathcal{T}_{h}$. We obtain in that way a nonlinear system in the unknowns $\underline{\sigma}_{h}, u_{h}$ and $\lambda_{h}$ where $\lambda_{h}$ is in some sense an approximation of $u$ along the edges of the triangulation $\mathcal{T}_{h}$. We next want to solve this nonlinear system by a simple Picard scheme by replacing the non linear term $\int_{\Omega} \underline{A}\left(\underline{\sigma}_{h}\right) \cdot \underline{\tau}_{h} d x$ by $\int_{\Omega}\left|\underline{\sigma}_{h}^{m}\right|^{q-2} \underline{\sigma}_{h}^{m+1} \cdot \underline{\tau}_{h} d x$. Introducing the "generalized barycenter" of $K$ :

$$
\underline{x}_{K}^{m}=\frac{\int_{K}\left|\underline{\sigma}_{h}^{m}(\underline{x})\right|^{q-2} \underline{x} d x}{\int_{K}\left|\underline{\sigma}_{h}^{m}(\underline{x})\right|^{q-2} d x} \in K
$$

and writing the lowest degree Raviart-Thomas vectorfield on $K \underline{\sigma}_{K}^{m+1}=\underline{\sigma}_{h}^{m+1}{ }_{\mid K}$, $K \in \mathcal{T}_{h}$, in the form

$$
\underline{\sigma}_{K}^{m+1}(\underline{x})=\underline{a}_{K}^{m+1}+c_{K}^{m+1}\left(\underline{x}-\underline{x}_{K}^{m}\right), \forall \underline{x} \in K,
$$

where $\underline{a}_{K}^{m+1} \in \mathbb{R}^{2}$ and $c_{K}^{m+1} \in \mathbb{R}$, we obtain the following expressions of $\underline{\sigma}_{K}^{m+1}$ and $u_{K}^{m+1}=u_{h}^{m+1}{ }_{\mid K}$ in terms of the Lagrange multiplier $\lambda_{h}^{m+1}{ }_{\mid \partial K}$ :

$$
\begin{aligned}
\underline{\sigma}_{K}^{m+1}(\underline{x}) & =\frac{\int_{\partial K} \lambda_{h}^{m+1} \underline{n} \underline{X}_{K} d s}{\int_{K}\left|\underline{\sigma}_{K}^{m}\right|^{q-2} d x}-\left(\frac{1}{2|K|} \int_{K} f d x\right)\left(\underline{x}-\underline{x}_{K}^{m}\right) \quad \forall \underline{x} \in K, \\
u_{K}^{m+1} & =\frac{1}{4|K|^{2}} \int_{K} f d x\left(\int_{K}\left|\underline{\sigma}_{K}^{m}(\underline{x})\right|^{q-2}\left|\underline{x}-\underline{x}_{K}^{m}\right|^{2} d x\right) \\
& +\frac{1}{2|K|} \int_{\partial K} \lambda_{h}^{m+1}\left(\underline{x}-\underline{x}_{K}^{m}\right) \cdot \underline{n}_{K} d s,
\end{aligned}
$$

$\underline{n}_{K}$ denoting the unitary normal vectorfield along the boundary of $K$. Expressing the continuity of the normal component of the vectorfield $\underline{\sigma}_{h}^{m+1}$ at each interface (i.e. at each interior edge $e$ ) of the triangulation $\mathcal{T}_{h}$, and applying the Picard scheme, we obtain at step $m+1$ a linear system in the unknowns $\lambda_{e}^{m+1}=\lambda_{h}^{m+1}{ }_{\mid e}, e$ running over the set of all interior edges of the triangulation $\mathcal{T}_{h}$ of the polygonal domain $\Omega$. Once $\lambda_{h}^{m+1}$ has been calculated, we then use it in formulas (0.4) and (0.5) in order to obtain $\underline{\sigma}_{h}^{m+1}$ and $u_{h}^{m+1}$, and continue the iterations.

Finally, in section 3 , we present a numerical test in the square $\Omega=]-1 / 2 ; 1 / 2\left[^{2}\right.$. Denoting by $r(\underline{x})=r\left(x_{1}, x_{2}\right)$ the distance to the origin, we consider the bounded function $u$ defined in the square by

$$
u(\underline{x})= \begin{cases}\left(\frac{1}{4}-r^{2}(\underline{x})\right)^{2} e^{-r^{2}(\underline{x}) / \varepsilon} & \text { if } r(\underline{x}) \leq \frac{1}{2}, \\ 0 \quad \text { if } r(\underline{x})>\frac{1}{2} .\end{cases}
$$


$f=-\operatorname{div}\left(|\underline{\nabla} u|^{p-2} \underline{\nabla} u\right)$ is easily computed and shown to be equal to:

$$
\begin{aligned}
f(\underline{x}) & =-2^{p-1} g^{p-2}(\underline{x})\left(2+\frac{1}{\varepsilon} g(\underline{x})\right)^{p-2} e^{-(p-1) \frac{r^{2}(\underline{x})}{\varepsilon}} r^{p-2}(\underline{x}) \\
& \times\left[2(p-1)\left(2+\frac{1}{\varepsilon} g(\underline{x})\right) r^{2}(\underline{x})+2(p-1) g(\underline{x})\left(3+\frac{1}{\varepsilon} g(\underline{x})\right) \frac{r^{2}(\underline{x})}{\varepsilon}\right. \\
& \left.-p g(\underline{x})\left(2+\frac{1}{\varepsilon} g(\underline{x})\right)\right],
\end{aligned}
$$

where

$$
g(\underline{x})=\frac{1}{4}-r^{2}(\underline{x}) .
$$

Ones verifies that $f \in L^{q}(\Omega)$ if the conjugate $p>\frac{1+\sqrt{5}}{2}=1.618 \ldots$ We test this example for $p=1.8$ and $p=3$. For each of these two cases, four graphics are given: $\left\|\underline{\sigma}-\underline{\sigma}_{h}\right\|_{0, q, \Omega},\left\|u-u_{h}\right\|_{0, p, \Omega}$, the ratio between $\| \underline{\sigma}-\left.\underline{\sigma}_{h}\right|_{0, q, \Omega}$ and the estimated error on $\underline{\sigma}_{h}$ and finally the ratio between $\left\|u-u_{h}\right\|_{0, p, \Omega}$ and the estimated error on $u_{h}$, all with respect to the number of degrees of freedom. One verifies the predicted order of convergence given respectively by theorem 3.2 for $p=1.8$ and theorem 3.1 for $p=3$ of Farhloul and Manouzi [1] and that the ratios between the errors and the estimated errors are asymptotically independent of the number of degrees of freedom.

\section{Definition of residues and bounds of the errors}

Let us first recall the weak formulation of the mixed method for the $p$-Laplacian introduced by Farhloul and Manouzi in [1]. Let $M$ denotes the Banach space $L^{p}(\Omega)$ $(1<p<+\infty)$ and $X$ the following:

$$
X=\left\{\underline{\tau} \in\left[L^{q}(\Omega)\right]^{2} ; \operatorname{div} \underline{\tau} \in L^{q}(\Omega)\right\},
$$

where $q$ denotes the harmonic conjugate of $p$. Endowed with the norm

$$
\|\underline{\tau}\|_{X}=\left(\|\underline{\tau}\|_{0, q, \Omega}^{q}+\|\operatorname{div} \underline{\tau}\|_{0, q, \Omega}^{q}\right)^{\frac{1}{q}},
$$

$X$ is also a Banach space. For every $\underline{\tau} \in\left[L^{q}(\Omega)\right]^{2}$, let us set $\underline{A}(\underline{\tau})=|\underline{\tau}|^{q-2} \underline{\tau}$. It is straightforward to check that when $\underline{\tau} \in\left[L^{q}(\Omega)\right]^{2}, \underline{A}(\underline{\tau}) \in\left[L^{p}(\Omega)\right]^{2}$. The weak formulation of the mixed method related to the Dirichlet problem for the $p$ Laplacian (0.1) reads as follows [1]: Find $(\underline{\sigma}, u) \in X \times M$ such that the following equations are satisfied:

$$
\begin{cases}\int_{\Omega} \underline{A}(\underline{\sigma}) \cdot \underline{\tau} d x+\int_{\Omega} u \operatorname{div} \underline{\tau} d x=0 & \forall \underline{\tau} \in X \\ \int_{\Omega}(\operatorname{div} \underline{\sigma}) v d x+\int_{\Omega} f v d x \quad=0 & \forall v \in M\end{cases}
$$


It is proved in [1] (theorem 2.1) that (1.10) possesses one and only one solution $(\underline{\sigma}, u)$ and that $u$ is the weak solution of $(0.1)$ and $\underline{\sigma}=|\underline{\nabla} u|^{p-2} \underline{\nabla} u$. Now let us consider a regular family of triangulations $\left(\mathcal{T}_{h}\right)_{h>0}$ on $\bar{\Omega}$ [4]. The following finite element spaces are defined in [1]:

$$
\begin{aligned}
& X_{h}=\left\{\underline{\tau}_{h} \in X ; \underline{\tau}_{h \mid K} \in R T_{0}(K), \forall K \in \mathcal{T}_{h}\right\}, \\
& M_{h}=\left\{v_{h} \in M ; v_{h \mid K} \in P_{0}(K), \forall K \in \mathcal{T}_{h}\right\},
\end{aligned}
$$

where $P_{0}(K)$ denotes the space of constant functions on the triangle $K$ and $[2,3]$ :

$$
R T_{0}(K)=P_{0}(K)^{2} \oplus P_{0}(K) \underline{x}, \quad \underline{x}=\left(x_{1}, x_{2}\right) .
$$

Then the finite element approximation to problem (1.10) is given by [1]: Find $\left(\underline{\sigma}_{h}, u_{h}\right) \in X_{h} \times M_{h}$ satisfying

$$
\begin{cases}\int_{\Omega} \underline{A}\left(\underline{\sigma}_{h}\right) \cdot \underline{\tau}_{h} d x+\int_{\Omega} u_{h} \operatorname{div} \underline{\tau}_{h} d x=0, & \forall \underline{\tau}_{h} \in X_{h} \\ \int_{\Omega}\left(\operatorname{div} \underline{\sigma}_{h}\right) v_{h} d x+\int_{\Omega} f v_{h} d x \quad=0, & \forall v_{h} \in M_{h}\end{cases}
$$

The existence and uniqueness of the solution of the discrete problem (1.14) follows from the uniform inf-sup condition [1]. A priori error estimates are also proved in Farhloul and Manouzi [1]. Now, we define two residues $R$ on $X$ and $r$ on $M$ by:

$$
\begin{aligned}
<R, \underline{\tau}> & =\left(\underline{A}\left(\underline{\sigma}_{h}\right), \underline{\tau}\right)+\left(\operatorname{div} \underline{\tau}, u_{h}\right), \quad \forall \underline{\tau} \in X, \\
<r, v> & =\left(\operatorname{div} \underline{\sigma}_{h}, v\right)+(f, v), \quad \forall v \in M .
\end{aligned}
$$

$R$ is a continuous linear form on $X$ and $r$ is a continuous linear form on $M$. When we will speak of $\|R\|$ (respectively $\|r\|$ ), it will mean the norm of $R$ (respectively $r$ ) as a continuous linear form on $X$ (respectively $M$ ). We have the following bound of $\left(\underline{A}\left(\underline{\sigma}_{h}\right)-\underline{A}(\underline{\sigma}), \underline{\sigma}_{h}-\underline{\sigma}\right)$ in terms of the norms of these two residues:

\section{Proposition 1.1.}

$$
\begin{aligned}
\left(\underline{A}\left(\underline{\sigma}_{h}\right)-\underline{A}(\underline{\sigma}), \underline{\sigma}_{h}-\underline{\sigma}\right) & \lesssim\|R\|\left\|\underline{\sigma}_{h}-\underline{\sigma}\right\|_{0, q, \Omega} \\
& +\|r\| \sup _{\underline{\tau} \in X} \frac{(\underline{A}(\underline{\sigma})-\underline{A}(\underline{\sigma} h), \underline{\tau})}{\|\underline{\tau}\| X}+2\|r\|\|R\|
\end{aligned}
$$

Before proceeding to the proof, let us make the following remark:

Remark 1.1. For $a, b \in \mathbb{R}_{+}$, we use the notation $a \lesssim b$ to mean that there exists a positive constant $C$ such that $a \leqslant C b$. Similarly, we write $a \sim b$ to mean that there exists two strictly positive constants $C_{1}$ and $C_{2}$ such that $C_{1} b \leq a \leq C_{2} b$.

Proof. Using the definition of $R(1.15)$ and the equation (1.10 (i)), we obtain:

$$
\left(\underline{A}\left(\underline{\sigma}_{h}\right)-\underline{A}(\underline{\sigma}), \underline{\tau}\right)+\left(\operatorname{div} \underline{\tau}, u_{h}-u\right)=<R, \underline{\tau}>, \quad \forall \underline{\tau} \in X .
$$


Substracting (1.10 (ii)) form (1.16), we obtain:

$$
\left(\operatorname{div}\left(\underline{\sigma}_{h}\right)-\operatorname{div}(\underline{\sigma}), v\right)=<r, v>, \quad \forall v \in M .
$$

Substracting (1.19) with $v=u_{h}-u$ from (1.18) with $\underline{\tau}=\underline{\sigma} h-\underline{\sigma}$, we obtain:

$$
\left(\underline{A}\left(\underline{\sigma}_{h}\right)-\underline{A}(\underline{\sigma}), \underline{\sigma}_{h}-\underline{\sigma}\right)=<R, \underline{\sigma}_{h}-\underline{\sigma}>-<r, u_{h}-u>.
$$

By the inf-sup condition stated in proposition 2.1 of [1] and (1.18) it follows:

$$
\begin{aligned}
\left\|u_{h}-u\right\|_{M} & \lesssim \sup _{\underline{\tau} \in X} \frac{\left(\operatorname{div} \underline{\tau}, u_{h}-u\right)}{\|\underline{\tau}\|_{X}} \\
& \lesssim \sup _{\underline{\tau} \in X} \frac{<R, \underline{\tau}>}{\|\underline{\tau}\| X}+\sup _{\underline{\tau} \in X} \frac{\left(\underline{A}(\underline{\sigma})-\underline{A}\left(\underline{\sigma}_{h}\right), \underline{\tau}\right)}{\|\underline{\tau}\|_{X}} \\
& =\|R\|+\sup _{\underline{\tau} \in X} \frac{\left(\underline{A}(\underline{\sigma})-\underline{A}\left(\underline{\sigma}_{h}\right), \underline{\tau}\right)}{\|\underline{\tau}\|_{X}} .
\end{aligned}
$$

From (1.20) and (1.21) it follows that:

$$
\begin{aligned}
\left(\underline{A}\left(\underline{\sigma}_{h}\right)-\underline{A}(\underline{\sigma}), \underline{\sigma}_{h}-\underline{\sigma}\right) & \lesssim\|R\|\left\|\underline{\sigma}_{h}-\underline{\sigma}\right\|_{X}+\|r\|\|R\| \\
& +\|r\| \sup _{\underline{\tau} \in X} \frac{(\underline{A}(\underline{\sigma})-\underline{A}(\underline{\sigma} h), \underline{\tau})}{\|\underline{\tau}\|_{X}}
\end{aligned}
$$

But:

$$
\begin{aligned}
\| \underline{\sigma}_{h}-\underline{\sigma}_{X} & \sim\left\|\underline{\sigma}_{h}-\underline{\sigma}\right\|_{0, q, \Omega}+\left\|\operatorname{div}\left(\underline{\sigma}_{h}-\underline{\sigma}\right)\right\|_{0, q, \Omega} \\
& =\left\|\underline{\sigma}_{h}-\underline{\sigma}\right\|_{0, q, \Omega}+\sup _{v \in L^{p}(\Omega)} \frac{<\operatorname{div}\left(\underline{\sigma}_{h}-\underline{\sigma}\right), v>}{\|v\|_{0, p, \Omega}} \\
& =\left\|\underline{\sigma}_{h}-\underline{\sigma}\right\|_{0, q, \Omega}+\|r\|
\end{aligned}
$$

by (1.19). The result follows now from (1.22) and (1.23).

Our purpose now is to bound the norms of the errors $\left\|\underline{\sigma}_{h}-\underline{\sigma}\right\|_{0, q, \Omega}$ and $\| u_{h}-$ $u \|_{0, p, \Omega}$ in terms of the norms of the residues $\|R\|$ and $\|r\|$. We will have to distinct two cases: $p \geq 2$ and $1<p<2$. We begin with the case $p \geq 2$.

Firstly, we need the following inequality due to Sandri [5] (see also [1] p. 74):

Lemma 1.1. We suppose $p \geq 2$. Then:

$$
\begin{aligned}
\left(\underline{A}\left(\underline{\sigma}_{h}\right)-\underline{A}(\underline{\sigma}), \underline{\sigma}_{h}-\underline{\sigma}\right) & \gtrsim \frac{\left\|\underline{\sigma}_{h}-\underline{\sigma}\right\|_{0, q, \Omega}^{2}}{\left\|\underline{\sigma}_{h}\right\|_{0, q, \Omega}^{2-q}+\|\underline{\sigma}\|_{0, q, \Omega}^{2-q}} \\
& +\int_{\Omega}\left|\underline{A}\left(\underline{\sigma}_{h}\right)-\underline{A}(\underline{\sigma})\right|\left|\underline{\sigma}_{h}-\underline{\sigma}\right| d x .
\end{aligned}
$$

We will also need the following inequality:

Lemma 1.2. Supposing $p \geq 2$, we have $\forall \underline{\tau} \in X$ :

$$
\left(\underline{A}(\underline{\sigma})-\underline{A}\left(\underline{\sigma}_{h}\right), \underline{\tau}\right) \lesssim\left[\int_{\Omega}\left|\underline{A}\left(\underline{\sigma}_{h}\right)-\underline{A}(\underline{\sigma})\right|\left|\underline{\sigma}_{h}-\underline{\sigma}\right| d x\right]^{\frac{1}{p}}\|\underline{\tau}\|_{0, q, \Omega}
$$


the multiplicative constant hidden in the sign $\lesssim$ being independent of $\underline{\tau}$.

The proof of inequality (1.25) relies on the following technical lemma:

\section{Lemma 1.3.}

$$
\forall p \geq 2, \forall \underline{x}, \underline{y} \in \mathbb{R}^{2},\left.|| \underline{x}\right|^{q-2} \underline{x}-\left.|\underline{y}|^{q-2} \underline{y}\right|^{p-1} \lesssim|\underline{x}-\underline{y}| .
$$

\section{Proof.}

(1) Let us first suppose that $|\underline{x}|=|\underline{y}|=1$. In that particular case, inequality (1.26) reduces to $|\underline{x}-\underline{y}|^{p-1} \lesssim|\underline{x}-\underline{y}|$. By hypothesis $p \geq 2$, which implies that $p-2 \geq 0$. Let us set $\zeta=\frac{|\underline{x}-\underline{y}|}{2} \leq 1$ which implies $\ln (\zeta) \leq 0$. Thus $\zeta^{p-2}=e^{(p-2) \ln (\zeta)} \leq 1$, implying that $\zeta^{p-1} \leq \zeta$. Recalling the definition of $\zeta$, it follows that $|\underline{x}-\underline{y}|^{p-1} \leq 2^{p-2}|\underline{x}-\underline{y}|$, i.e. $|\underline{x}-\underline{y}|^{p-1} \lesssim|\underline{x}-\underline{y}|$ establishing inequality (1.26) in the particular case $|\underline{x}|=|\underline{y}|=\underline{=}$.

(2) One verifies easily that the left-hand side of inequality (1.26) is a homogeneous function of degree 1, i.e. that

$$
\left.|| t \underline{x}\right|^{q-2} t \underline{x}-\left.|t \underline{y}|^{q-2} t \underline{y}\right|^{p-1}=\left.t|| \underline{x}\right|^{q-2} \underline{x}-|\underline{y}|^{q-2} \underline{y} \mid
$$

for every $t>0$ which implies using the first step of our proof that inequality (1.26) is still true under the weaker condition that $|\underline{x}|=|\underline{y}|$.

(3) Thus it remains to prove inequality (1.26) when $|\underline{x}| \neq|\underline{y}| . \underline{x}$ and $y$ playing a symmetric role in inequality (1.26), we may suppose $|\underline{x}|>|\underline{y}|$. Considering $\underline{y}$ and $\lambda \underline{x}$ with $\lambda=\frac{|\underline{y}|}{|\underline{x}|}$ so that $|\lambda \underline{x}|=|\underline{y}|$, it follows by the previous step of our work that

$$
\left.|| \underline{y}\right|^{q-2} \underline{y}-\left.|\lambda \underline{x}|^{q-2} \lambda \underline{x}\right|^{p-1} \lesssim|\underline{y}-\lambda \underline{x}| .
$$

But

$$
\begin{aligned}
|\underline{y}-\lambda \underline{x}| & \leq|\underline{y}-\underline{x}|+|\underline{x}||1-\lambda| \\
& =|\underline{y}-\underline{x}|+|\underline{x}|\left|1-\frac{|\underline{y}|}{|\underline{x}|}\right| \\
& \leq 2|\underline{y}-\underline{x}| .
\end{aligned}
$$

From (1.27) and (1.28), it follows that

$$
\left.|| \underline{y}\right|^{q-2} \underline{y}-\left.|\lambda \underline{x}|^{q-2} \lambda \underline{x}\right|^{p-1} \lesssim|\underline{y}-\underline{x}| .
$$

On the other hand:

$$
\begin{aligned}
\left.|| \lambda \underline{x}\right|^{q-2} \lambda \underline{x}-\left.|\underline{x}|^{q-2} \underline{x}\right|^{p-1} & =\left.\left.\left|\left(\left(\frac{|\underline{y}|}{|\underline{x}|}\right)^{q-1}-1\right)\right| \underline{x}\right|^{q-2} \underline{x}\right|^{p-1} \\
& =\left.|| \underline{y}\right|^{q-1}-\left.|\underline{x}|^{q-1}\right|^{p-1} .
\end{aligned}
$$


But it is elementary to prove that for $s \geq 1$ and $\alpha \geq 0, \beta \geq 0$ :

$$
\left|\alpha^{1 / s}-\beta^{1 / s}\right|^{s} \leq|\alpha-\beta| .
$$

Applying (1.31) with $\alpha=|\underline{y}|, \beta=|\underline{x}|$ and $s=p-1$, we get:

$$
\left.|| \underline{y}\right|^{q-1}-\left.|\underline{x}|^{q-1}\right|^{p-1} \leq|| \underline{y}|-| \underline{x}|| \leq|\underline{y}-\underline{x}| .
$$

From (1.30) and (1.32), it follows that:

$$
\left.|| \lambda \underline{x}\right|^{q-2} \lambda \underline{x}-\left.|\underline{x}|^{q-2} \underline{x}\right|^{p-1} \leq|\underline{x}-\underline{y}| .
$$

From (1.29) and (1.33) we obtain:

$$
\begin{aligned}
\left.|| \underline{y}\right|^{q-2} \underline{y}-\left.|\underline{x}|^{q-2} \underline{x}\right|^{p-1} & \leq 2^{p-1}\left(\left.|| \underline{y}\right|^{q-2} \underline{y}-\left.|\lambda \underline{x}|^{q-2} \lambda \underline{x}\right|^{p-1}+\left.|| \lambda \underline{x}\right|^{q-2} \lambda \underline{x}-\left.|\underline{x}|^{q-2} \underline{x}\right|^{p-1}\right) \\
& \lesssim|\underline{x}-\underline{y}|,
\end{aligned}
$$

what was to be proved.

Now we give the proof of lemma 1.2:

\section{Proof.}

$$
\begin{aligned}
\left(\underline{A}(\underline{\sigma})-\underline{A}\left(\underline{\sigma}_{h}\right), \underline{\tau}\right) & \lesssim\left(\int_{\Omega}\left|\underline{A}(\underline{\sigma})-\underline{A}\left(\underline{\sigma}_{h}\right)\right|^{p} d x\right)^{1 / p}\|\underline{\tau}\|_{0, q, \Omega} \\
& =\left(\left.\int_{\Omega}|| \underline{\sigma}\right|^{q-2} \underline{\sigma}-\left.|\underline{\sigma} h|^{q-2} \underline{\sigma}_{h}\right|^{p} d x\right)^{1 / p}\|\underline{\tau}\|_{0, q, \Omega} \\
& =\left(\left.\int_{\Omega}|| \underline{\sigma}\right|^{q-2} \underline{\sigma}-\left.\left|\underline{\sigma}_{h}\right|^{q-2} \underline{\sigma}_{h}\right|^{p-1}\left|\underline{A}(\underline{\sigma})-\underline{A}\left(\underline{\sigma}_{h}\right)\right| d x\right)^{1 / p}\|\underline{\tau}\|_{0, q, \Omega} \\
& \lesssim\left[\int_{\Omega}\left|\underline{\sigma}-\underline{\sigma}_{h}\right| \underline{A}(\underline{\sigma})-\underline{A}\left(\underline{\sigma}_{h}\right) \mid d x\right]^{1 / p}\|\underline{\tau}\|_{0, q, \Omega}
\end{aligned}
$$

due to lemma 1.3. This proves the result.

Using lemma 1.1, 1.2 and inequality (1.22), we are now in a position to prove the following result:

Proposition 1.2. Supposing $p \geq 2$, we have the following bounds in terms of the norms of the residues:

$$
\left\|\underline{\sigma}_{h}-\underline{\sigma}\right\|_{0, q, \Omega}^{2} \lesssim\|R\|^{2}+\|r\|^{q}+\|r\|\|R\|
$$

and

$$
\int_{\Omega}\left|\underline{A}\left(\underline{\sigma}_{h}\right)-\underline{A}(\underline{\sigma})\right|\left|\underline{\sigma}_{h}-\underline{\sigma}\right| d x \lesssim\|R\|^{2}+\|r\|^{q}+\|r\|\|R\| .
$$


Proof. Reading inequality (1.24) from right to left and using inequality (1.17), we obtain:

$$
\begin{aligned}
& \frac{\left\|\underline{\sigma}_{h}-\underline{\sigma}\right\|_{0, q, \Omega}^{2}}{\left\|\underline{\sigma}_{h}\right\|_{0, q, \Omega}^{2-q}+\|\underline{\sigma}\|_{0, q, \Omega}^{2-q}}+\int_{\Omega}\left|\underline{A}\left(\underline{\sigma}_{h}\right)-\underline{A}(\underline{\sigma})\right|\left|\underline{\sigma}_{h}-\underline{\sigma}\right| d x \\
\lesssim & \|R\|\left\|\underline{\sigma}_{h}-\underline{\sigma}\right\|_{0, q, \Omega}+\|r\| \sup _{\underline{\tau} \in X} \frac{(\underline{A}(\underline{\sigma})-\underline{A}(\underline{\sigma} h), \underline{\tau})}{\|\underline{\tau}\| X}+2\|r\|\|R\| \\
\lesssim & \|R\|\left\|\underline{\sigma}_{h}-\underline{\sigma}\right\|_{0, q, \Omega}+\|r\|\left[\int_{\Omega}\left|\underline{A}\left(\underline{\sigma}_{h}\right)-\underline{A}(\underline{\sigma})\right||\underline{\sigma} h-\underline{\sigma}| d x\right]^{1 / p}+2\|r\|\|R\|
\end{aligned}
$$

by lemma 1.2. Now using Young's inequality, i.e. $\forall a \geq 0, \quad \forall b \geq 0, \quad a b \leq \frac{1}{p} a^{p}+\frac{1}{q} b^{q}$, we obtain $\forall \varepsilon>0$ and $\forall \bar{\varepsilon}>0$ from the previous inequality:

$$
\begin{array}{r}
\frac{\left\|\underline{\sigma}_{h}-\underline{\sigma}\right\|_{0, q, \Omega}^{2}}{\|\underline{\sigma} h\|_{0, q, \Omega}^{2-q}+\|\underline{\sigma}\|_{0, q, \Omega}^{2-q}}+\int_{\Omega}\left|\underline{A}\left(\underline{\sigma}_{h}\right)-\underline{A}(\underline{\sigma})\right||\underline{\sigma} h-\underline{\sigma}| d x \\
\lesssim \varepsilon^{-1}\left(\|\underline{\sigma} h\|_{0, q, \Omega}^{2-q}+\|\underline{\sigma}\|_{0, q, \Omega}^{2-q}\right)\|R\|^{2}+\varepsilon \frac{\|\underline{\sigma} h-\underline{\sigma}\|_{0, q, \Omega}^{2}}{\|\underline{\sigma} h\|_{0, q, \Omega}^{2-q}+\|\underline{\sigma}\|_{0, q, \Omega}^{2-q}} \\
+(\bar{\varepsilon})^{-q}\|r\|^{q}+(\bar{\varepsilon})^{p} \int_{\Omega}\left|\underline{A}\left(\underline{\sigma}_{h}\right)-\underline{A}(\underline{\sigma})\right|\left|\underline{\sigma}_{h}-\underline{\sigma}\right| d x+\|r\|\|R\| .
\end{array}
$$

Choosing adequately $\varepsilon>0$ and $\bar{\varepsilon}>0$, it follows from the previous inequality the two following ones:

$$
\begin{aligned}
\left\|\underline{\sigma}_{h}-\underline{\sigma}\right\|_{0, q, \Omega}^{2} & \lesssim\left(\left\|\underline{\sigma}_{h}\right\|_{0, q, \Omega}^{2-q}+\|\underline{\sigma}\|_{0, q, \Omega}^{2-q}\right) \\
& \times\left\{\left(\left\|\underline{\sigma}_{h}\right\|_{0, q, \Omega}^{2-q}+\|\underline{\sigma}\|_{0, q, \Omega}^{2-q}\right)\|R\|^{2}+\|r\|^{q}+\|r\|\|R\|\right\}
\end{aligned}
$$

and

$\int_{\Omega}\left|\underline{A}\left(\underline{\sigma}_{h}\right)-\underline{A}(\underline{\sigma})\right|\left|\underline{\sigma}_{h}-\underline{\sigma}\right| d x \lesssim\left(\left\|\underline{\sigma}_{h}\right\|_{0, q, \Omega}^{2-q}+\|\underline{\sigma}\|_{0, q, \Omega}^{2-q}\right)\|R\|^{2}+\|r\|^{q}+\|r\|\|R\|$.

On the other hand, following the proof of theorem 2.1 of [6] (see also theorem 2.1 of $[1])$, we have

$$
\|\underline{\sigma}\|_{X} \leq\|f\|_{0, q, \Omega} \text { and }\left\|\underline{\sigma}_{h}\right\|_{X} \leq\|f\|_{0, q, \Omega} .
$$

Using this fact in the right-hand side of (1.39) (respectively (1.40)), we obtain the inequality (1.35) (respectively (1.36)).

From (1.21), lemma 1.2 and (1.36), it follows that:

Corollary 1.1. Supposing $p \geq 2$, we have the following bound for the norm of the error $u-u_{h}$ in terms of the norms of the residues:

$$
\left\|u_{h}-u\right\|_{0, p, \Omega} \lesssim\|R\|+\left(\|R\|^{2}+\|r\|^{q}+\|r\|\|R\|\right)^{1 / p} .
$$

We now turn to the case $1<p<2$. Firstly, we are going to bound the norm of the error on $\underline{\sigma}_{h}$, i.e. $\left\|\underline{\sigma}_{h}-\underline{\sigma}\right\|_{0, q, \Omega}$. 
Proposition 1.3. Supposing $1<p<2$, we have the following bound on the norm of the error on $\underline{\sigma}_{h}$ :

$$
\left\|\underline{\sigma}_{h}-\underline{\sigma}\right\|_{0, q, \Omega} \lesssim\|R\|^{p / q}+\|r\|^{2 / q}+(\|r\|\|R\|)^{1 / q} .
$$

Proof. By inequality (3.14) of [1] (see also Sandri [5]), we have:

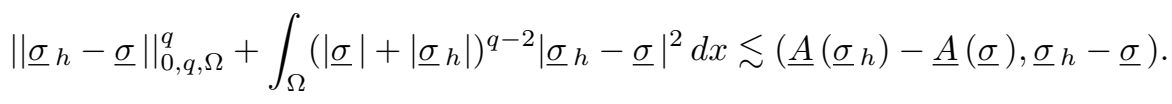

Using inequality (1.17) from proposition 1.1, it follows from the previous inequality

$$
\begin{array}{r}
\left\|\underline{\sigma}_{h}-\underline{\sigma}\right\|_{0, q, \Omega}^{q}+\int_{\Omega}\left(|\underline{\sigma}|+\left|\underline{\sigma}_{h}\right|\right)^{q-2}\left|\underline{\sigma}_{h}-\underline{\sigma}\right|^{2} d x \\
\lesssim\|R\|\left\|\underline{\sigma}_{h}-\underline{\sigma}\right\|_{0, q, \Omega}+\|r\|_{\sup _{\underline{\tau}} \in} \frac{\left(\underline{A}(\underline{\sigma})-\underline{A}\left(\underline{\sigma}_{h}\right), \underline{\tau}\right)}{\|\underline{\tau}\|_{X}}+2\|r\|\|R\| .
\end{array}
$$

By inequality (3.15) of [1],

$$
\left\|\underline{A}(\underline{\sigma})-\underline{A}\left(\underline{\sigma}_{h}\right)\right\|_{0, p, \Omega} \lesssim\left[\int_{\Omega}\left(|\underline{\sigma}|+\left|\underline{\sigma}_{h}\right|\right)^{q-2}\left|\underline{\sigma}_{h}-\underline{\sigma}\right|^{2} d x\right]^{1 / 2}\left[\|\underline{\sigma}\|_{0, q, \Omega}+\left\|\underline{\sigma}_{h}\right\|_{0, q, \Omega}\right]^{\frac{q-2}{2}},
$$

and Young's inequality, we obtain from inequality (1.45) that, $\forall \varepsilon>0$ and $\forall \bar{\varepsilon}>0$,

$$
\begin{array}{r}
\left\|\underline{\sigma}_{h}-\underline{\sigma}\right\|_{0, q, \Omega}^{q}+\int_{\Omega}\left(|\underline{\sigma}|+\left|\underline{\sigma}_{h}\right|\right)^{q-2}\left|\underline{\sigma}_{h}-\underline{\sigma}\right|^{2} d x \\
\lesssim \varepsilon^{q}\left\|\underline{\sigma}_{h}-\underline{\sigma}\right\|_{0, q, \Omega}^{q}+\frac{1}{\varepsilon^{p}}\|R\|^{p}+\bar{\varepsilon} \int_{\Omega}\left(|\underline{\sigma}|+\left|\underline{\sigma}_{h}\right|\right)^{q-2}\left|\underline{\sigma}_{h}-\underline{\sigma}\right|^{2} d x \\
+\frac{1}{\bar{\varepsilon}}\left[\|\underline{\sigma}\|_{0, q, \Omega}+\left\|\underline{\sigma}_{h}\right\|_{0, q, \Omega}\right]^{q-2}\|r\|^{2}+\|r\|\|R\| .
\end{array}
$$

Choosing adequately $\varepsilon>0$ and $\bar{\varepsilon}>0$, il follows from the previous inequality the following one:

$$
\left\|\underline{\sigma}_{h}-\underline{\sigma}\right\|_{0, q, \Omega}^{q} \lesssim\|R\|^{p}+\left[\|\underline{\sigma}\|_{0, q, \Omega}+\left\|\underline{\sigma}_{h}\right\|_{0, q, \Omega}\right]^{q-2}\|r\|^{2}+\|r\|\|R\| .
$$

Using (1.41), it follows that

$$
\left\|\underline{\sigma}_{h}-\underline{\sigma}\right\|_{0, q, \Omega}^{q} \lesssim\|R\|^{p}+\|r\|^{2}+\|r\|\|R\|,
$$

and taking the two-sides of this inequality to the power $\frac{1}{q}$, we obtain the result.

Proposition 1.4. Supposing $1<p<2$, we have the following bound on the norm of the error on $u_{h}$ :

$$
\left\|u-u_{h}\right\|_{0, p, \Omega} \lesssim\|R\|+\|r\|+\|R\|^{p / 2} .
$$

Proof. By the definition of $R$ (see (1.15)) and equation (1.10 (i)), we have

$$
\left(\underline{A}\left(\underline{\sigma}_{h}\right)-\underline{A}(\underline{\sigma}), \underline{\tau}\right)+\left(\operatorname{div} \underline{\tau}, u_{h}-u\right)=<R, \underline{\tau}>, \quad \forall \underline{\tau} \in X .
$$


By the inf-sup inequality (cf. proposition 2.1 of [1]) and the previous equation, we have the following bound on $\left\|u_{h}-u\right\|_{0, p, \Omega}$ :

$$
\begin{aligned}
\left\|u_{h}-u\right\|_{0, p, \Omega} & \lesssim \sup _{\underline{\tau} \in X} \frac{\langle R, \underline{\tau}\rangle}{\|\underline{\tau}\|_{X}}+\sup _{\underline{\tau} \in X} \frac{\left(\underline{A}\left(\underline{\sigma}_{h}\right)-\underline{A}(\underline{\sigma}), \underline{\tau}\right)}{\|\underline{\tau}\|_{X}} \\
& \lesssim\|R\|+\left\|\underline{A}\left(\underline{\sigma}_{h}\right)-\underline{A}(\underline{\sigma})\right\|_{0, p, \Omega} .
\end{aligned}
$$

By inequality (3.15) of [1] and (1.41), it follows that

$$
\left\|\underline{A}\left(\underline{\sigma}_{h}\right)-\underline{A}(\underline{\sigma})\right\|_{0, p, \Omega} \lesssim\left[\int_{\Omega}\left(|\underline{\sigma}|+\left|\underline{\sigma}_{h}\right|\right)^{q-2}\left|\underline{\sigma}_{h}-\underline{\sigma}\right|^{2} d x\right]^{1 / 2} .
$$

On the other hand, inequality (1.46) implies

$$
\int_{\Omega}\left(|\underline{\sigma}|+\left|\underline{\sigma}_{h}\right|\right)^{q-2}\left|\underline{\sigma}_{h}-\underline{\sigma}\right|^{2} d x \lesssim\|R\|^{p}+\|r\|^{2}+\|r\|\|R\| .
$$

From (1.50), (1.51) and (1.52), it follows that

$$
\begin{aligned}
\left\|u-u_{h}\right\|_{0, p, \Omega} & \lesssim\|R\|+\left(\|R\|^{p}+\|r\|^{2}+\|r\|\|R\|\right)^{1 / 2} \\
& \lesssim\|R\|+\|R\|^{p / 2}+\|r\|+\|r\|^{1 / 2}\|R\|^{1 / 2} \\
& \lesssim\|r\|+\|R\|+\|R\|^{p / 2} .
\end{aligned}
$$

Now, in both cases $p \geq 2$ and $1<p<2$, we are reduced to bound the norms of the residues $R$ and $r$ in terms of computed or known quantities. This is immediate for $r$.

Proposition 1.5. $\|r\|=\eta_{2}$ where $\eta_{2}=\left(\sum_{K \in \mathcal{T}_{h}} \eta_{2}(K)^{q}\right)^{1 / q}$ and $\eta_{2}(K)=\left\|f-P_{h}^{0} f\right\|_{0, q, K}, \forall K \in \mathcal{T}_{h}$.

Proof. $r$ has been defined by equation (1.16) as being $\operatorname{div} \underline{\sigma}_{h}+f$. But by the second equation of the discretized mixed formulation (1.14), we have $\operatorname{div} \underline{\sigma}_{h}=-P_{h}^{0} f$, where $P_{h}^{0}$ denotes the projection operator defined by:

$$
\begin{aligned}
P_{h}^{0}: L^{q}(\Omega) & \longrightarrow M_{h} \\
v & \longmapsto P_{h}^{0} v,
\end{aligned}
$$

$P_{h}^{0} v$ being the function constant on each triangle $K$ equal to the mean of $v$ on $K$. Thus $r=f-P_{h}^{0} f$ and

$$
\|r\|=\sup _{v \in M \backslash\{0\}} \frac{<r, v>}{\|v\|_{M}}=\left\|f-P_{h}^{0} f\right\|_{L^{q}(\Omega)}=\left(\sum_{K \in \mathcal{T}_{h}} \eta_{2}(K)^{q}\right)^{1 / q} .
$$

We turn now, to the far more serious problem to bound the norm of $R$ which has been defined by equation (1.15). To do that we need the Helmholtz decomposition of arbitrary vectorfields $\underline{\tau} \in\left[L^{q}(\Omega)\right]^{2}$ with estimates. 
Theorem 1.1. Let $q \in] 1,+\infty\left[\right.$. For an arbitrary vectorfield $\underline{\tau} \in\left[L^{q}(\Omega)\right]^{2}$ such that $\operatorname{div} \underline{\tau} \in L^{q}(\Omega)$, there exists a function $z \in W^{2, q}(\Omega)$ and a function $\Phi \in W^{1, q}(\Omega)$ such that:

$$
\begin{aligned}
\underline{\tau} & =\underline{\nabla} z+\underline{\operatorname{curl}} \Phi \\
\|z\|_{W^{2, q}(\Omega)}+\|\Phi\|_{W^{1, q}(\Omega)} & \lesssim\|\underline{\tau}\|_{0, q, \Omega}+\|\operatorname{div} \underline{\tau}\|_{0, q, \Omega} .
\end{aligned}
$$

Proof. Let $D$ be a bounded domain of the plane, with a regular boundary and containing $\bar{\Omega}$. Let us denote by $(\operatorname{div} \underline{\tau})^{\sim}$ the extension of $\operatorname{div} \underline{\tau}$ to $D$ by 0 out of $\Omega$. Of course, $(\operatorname{div} \underline{\tau})^{\sim} \in L^{q}(D)$. In $D$, we consider the boundary value problem:

$$
\left\{\begin{array}{l}
\Delta S=(\operatorname{div} \underline{\tau})^{\sim} \text { in } \mathrm{D} \\
S_{\mid \partial D}=0 .
\end{array}\right.
$$

This problem possesses one and only one solution in $H_{0}^{1}(D)$. Moreover by theorem 2.4.2.5 of [7] p 124-125, the variational solution $S \in W^{2, q}(D)$. It follows from the closed graph theorem that also:

$$
\|S\|_{W^{2, q}(D)} \lesssim\left\|(\operatorname{div} \underline{\tau})^{\sim}\right\|_{0, q, D}=\|\operatorname{div} \underline{\tau}\|_{0, q, \Omega} .
$$

Let us set $z=S_{\mid \Omega}$. Consequently, we have:

$$
\|z\|_{W^{2, q}(\Omega)} \lesssim\|\operatorname{div} \underline{\tau}\|_{0, q, \Omega} .
$$

By (1.56), we have that $\operatorname{div}(\underline{\nabla} z)=\operatorname{div} \underline{\tau}$. Let us set:

$$
\underline{v}=\underline{\tau}-\underline{\nabla} z .
$$

$\underline{v}$ is a divergence-free vectorfield in $\Omega, \underline{v} \in\left[L^{q}(\Omega)\right]^{2}$ and

$$
\|\underline{v}\|_{0, q, \Omega} \lesssim\|\underline{\tau}\|_{0, q, \Omega}+\|\operatorname{div} \underline{\tau}\|_{0, q, \Omega}
$$

by inequality (1.58). We now suppose that $q \geq 2$ (the proof in the case $1<q<2$ will be done later). This hypothesis implies that $\underline{v} \in\left[L^{2}(\Omega)\right]^{2}$. This allows us to apply theorem I.3.1 p 37 of [8] which tells us that there exists one and only one function $\Phi \in H^{1}(\Omega) \cap L_{0}^{2}(\Omega)$ such that the divergence-free vectorfield $\underline{v}$ may be written in the form

$$
\underline{v}=\underline{\operatorname{curl}} \Phi .
$$

Obviously, $|\Phi|_{W^{1, q}(\Omega)}=\|\underline{v}\|_{0, q, \Omega}$ as $v_{1}=\frac{\partial \Phi}{\partial x_{2}}$ and $v_{2}=-\frac{\partial \Phi}{\partial x_{1}}$. Moreover by Sobolev's imbedding theorem and Poincaré's inequality for functions in $H^{1}(\Omega)$ of mean zero [9]:

$$
\left.\left\|\left.\Phi\right|_{0, q, \Omega} \lesssim\right\| \Phi\right|_{H^{1}(\Omega)} \lesssim|\Phi|_{H^{1}(\Omega)} \lesssim|\Phi|_{W^{1, q}(\Omega)} .
$$

Putting these two facts together, it follows that:

$$
\|\Phi\|_{W^{1, q}(\Omega)} \lesssim\|\underline{v}\|_{0, q, \Omega} .
$$


Using inequalities (1.62) and (1.60), we obtain that

$$
\|\Phi\|_{W^{1, q}(\Omega)} \lesssim\|\underline{\tau}\|_{0, q, \Omega}+\|\operatorname{div} \underline{\tau}\|_{0, q, \Omega} .
$$

Adding the two inequalities (1.58) and (1.63), we obtain (1.55). Also from the equalities (1.59) and (1.61) follows (1.54).

We now turn to the case $1<q<2$. The proof of this case requires the following density lemma.

Lemma 1.4. Let us suppose that $q \in] 1,2[$. The vectorial subspace $\{\underline{v} \in$ $\left.\left[L^{2}(\Omega)\right]^{2} ; \operatorname{div} \underline{v}=0\right\}$ is dense in the vectorial space $\{\underline{v} \in X ; \operatorname{div} \underline{v}=0\}$ for the norm of $X$.

Proof. By lemma 1.1.2 p. 16 of Mghazli's thesis [10], we know that $[D(\bar{\Omega})]^{2}$ is dense in $X$. Let $\underline{v} \in X$ such that $\operatorname{div} \underline{v}=0$ be given. There exists a sequence of vectorfields $\left(\underline{v}_{k}^{*}\right)_{k \geq 1} \subset[D(\bar{\Omega})]^{2}$ such that $\left(\underline{v}_{k}^{*}\right)_{k \geq 1}$ tends to $\underline{v}$ in the sense of the norm of $X$. Proceeding like in (1.56)-(1.58) but with the vectorfield $\underline{v}_{k}^{*}$ instead of $\underline{\tau}$ in the right-hand side of the first equation of (1.56), we construct a sequence $\left(z_{k}\right)_{k \geq 1} \subset W^{2, q}(\Omega)$ (theorem 2.4.2.5 of [7] p 124-125 that we have used is also true in the case $1<q<2$ ) such that

$$
\left\|z_{k}\right\|_{W^{2, q}(\Omega)} \lesssim\left\|\operatorname{div} \underline{v}_{k}^{*}\right\|_{0, q, \Omega}
$$

and $\operatorname{div}\left(\underline{\nabla} z_{k}\right)=\operatorname{div} \underline{v}_{k}^{*}, \quad \forall k \geq 1$. Let us set $\underline{v}_{k}=\underline{v}_{k}^{*}-\underline{\nabla} z_{k} \cdot \underline{v}_{k}$ is a square integrable vectorfield and also divergence-free. Thus $\underline{v}_{k}$ belongs to the vectorial subspace $\left\{\underline{v} \in\left[L^{2}(\Omega)\right]^{2} ; \operatorname{div} \underline{v}=0\right\}$. It remains to prove that the sequence $\left(\underline{v}_{k}\right)_{k \geq 1}$ tends to $\underline{v}$ in the sense of the norm of $X$.

$$
\left\|\underline{v}_{k}-\underline{v}\right\|_{X} \leq\left\|\underline{v}_{k}^{*}-\underline{v}\right\|_{X}+\left\|\underline{\nabla} z_{k}\right\|_{X} .
$$

$\underline{v}_{k}^{*}$ tends to $\underline{v}$ in the sense of the norm of $X$. On the other hand by inequality (1.64) :

$$
\left\|\underline{\nabla} z_{k}\right\|_{X} \lesssim\left\|\operatorname{div} \underline{v}_{k}^{*}\right\|_{0, q, \Omega}
$$

and as $\operatorname{div} \underline{v}_{k}^{*}$ tends to $\operatorname{div} \underline{v}=0$ in $L^{q}(\Omega)$ as $k \rightarrow+\infty$, it follows that $\left\|\underline{\nabla} z_{k}\right\|_{X} \rightarrow 0$ as $k \rightarrow+\infty$. From these two facts and inequality (1.65) it follows that $\|\underline{v} k-\underline{v}\|_{X} \rightarrow$ 0 as $k \rightarrow+\infty$.

We now turn back to the proof of theorem 1.1 when $1<q<2$.

(Proof of theorem 1.1 when $1<q<2$.)

By the preceding lemma, there exists a sequence $\left(\underline{v}_{k}\right)_{k \geq 1}$ of square-integrable divergence-free vectorfields on $\Omega$ converging to the vectorfield $\underline{v}$ defined by (1.59) in the sense of the norm of $X$. Applying theorem I.3.1 p. 37 of [8] to the vectorfield $\underline{v}_{k}$, there exists one and only one function $\phi_{k} \in H^{1}(\Omega) \cap L_{0}^{2}(\Omega)$ such that $\underline{\operatorname{curl}} \phi_{k}=\underline{v}_{k}$. As $\underline{v}_{k} \rightarrow \underline{v}$ in $\left[L^{q}(\Omega)\right]^{2}$, the sequence $\left(\underline{\nabla} \phi_{k}\right)_{k \geq 1}$ is a Cauchy sequence in $\left[L^{q}(\Omega)\right]^{2}$. 
Moreover, by a compacity argument similar to that used to prove lemma 1.8 in [11], it is easily seen that the mapping:

$$
W^{1, q}(\Omega) \rightarrow \mathbb{R}_{+}: \Psi \mapsto\left\|\frac{\partial \Psi}{\partial x_{1}}\right\|_{0, q, \Omega}+\left\|\frac{\partial \Psi}{\partial x_{2}}\right\|_{0, q, \Omega}+\left|\int_{\Omega} \Psi d x\right|
$$

is an equivalent norm on $W^{1, q}(\Omega)$. In view of (1.67) and the fact that the functions $\phi_{k}, \quad k \geq 1$, are all of mean 0 , it follows that $\left(\phi_{k}\right)_{k \geq 1}$ is a Cauchy sequence in $W^{1, q}(\Omega)$. Let us set $\Phi=\lim _{k \rightarrow+\infty} \phi_{k}$ in $W^{1, q}(\Omega)$. $\Phi$ is also of mean 0 and curl $\Phi=$ $\underline{v}$. By these facts and (1.67), it follows:

$$
\left\|\left.\Phi\right|_{W^{1, q}(\Omega)} \lesssim|\Phi|_{W^{1, q}(\Omega)}=\right\| \underline{v} \|_{0, q, \Omega} .
$$

Using (1.68), (1.60) and (1.58), we obtain inequality (1.55). By (1.59) and $\underline{v}=$ curl $\Phi$ follows (1.54).

Lemma 1.5. For every $\underline{\tau} \in X$ :

$$
\begin{aligned}
<R, \underline{\tau}> & =\sum_{K \in \mathcal{T}_{h}}\left(\underline{A}_{h}\left(\underline{\sigma}_{h}\right), \underline{\nabla} z-\pi_{h} \underline{\nabla} z\right) \\
& +\sum_{K \in \mathcal{T}_{h}}\left(\operatorname{curl}\left(\underline{\mathrm{A}}\left(\underline{\sigma}_{\mathrm{h}}\right)\right), \Phi-\mathrm{I}_{\mathrm{cl}}(\Phi)\right) \\
& -\sum_{E \in \mathcal{E}_{h}}<\llbracket \underline{A}\left(\underline{\sigma}_{h}\right) \cdot \underline{t} \rrbracket_{E}, \Phi-I_{c l}(\Phi)>_{E}
\end{aligned}
$$

where:

- $(z, \Phi) \in W^{2, q}(\Omega) \times W^{1, q}(\Omega)$ denotes the Helmholtz decomposition of the vectorfield $\underline{\tau} \in X$,

- $I_{c l}(\Phi)$ is the Clément interpolate of $\Phi$ (see [8] p. 109-111),

- $\underline{\sigma}_{h}$ is defined by (1.14),

- $\mathcal{E}_{h}$ denotes the set of all edges of the triangulation $\mathcal{T}_{h}$,

- $\left.\left[\left[\underline{A}\left(\underline{\sigma}_{h}\right)\right) \cdot \underline{t}\right]\right]_{E}$ denotes the tangential jump of $\underline{A}\left(\underline{\sigma}_{h}\right)$,

- $\pi_{h} \underline{\nabla} z$ is the Raviart-Thomas interpolate of lowest degree of $\underline{\nabla} z$.

Proof. By (1.15) for every $\underline{\tau} \in X$,

$$
<R, \underline{\tau}>=\left(\underline{A}\left(\underline{\sigma}_{h}\right), \underline{\tau}\right)+\left(\operatorname{div} \underline{\tau}, u_{h}\right) .
$$

By theorem 1.1, for every $\underline{\tau} \in X$, there exists $(z, \Phi) \in W^{2, q}(\Omega) \times W^{1, q}(\Omega)$ such that $\underline{\tau}=\underline{\nabla} z+\underline{\text { curl }} \Phi$ and the estimates (1.55) holds. Using this Helmholtz decomposition, (1.70) may be rewritten:

$$
\left.\left.<R, \underline{\tau}>=\left(\underline{A}^{(\sigma} \underline{\sigma}_{h}\right), \underline{\nabla} z\right)+\left(\underline{A}^{(\sigma} \underline{\sigma}_{h}\right), \underline{\operatorname{curl}} \Phi\right)+\left(\operatorname{div}(\underline{\nabla} z), u_{h}\right) .
$$

Now, $\underline{\nabla} z \in\left[W^{1, q}(\Omega)\right]^{2}$ and thus its Raviart-Thomas interpolate of the lowest degree is well defined (see (14) of [11]). Let us denote it $\pi_{h}(\underline{\nabla} z)$. We have:

$$
\left(\operatorname{div} \pi_{h}(\underline{\nabla} z), v_{h}\right)=\left(\operatorname{div}(\underline{\nabla} z), v_{h}\right), \forall v_{h} \in M_{h} .
$$


Thus (1.71) may be rewritten:

$$
<R, \underline{\tau}>=(\underline{A}(\underline{\sigma} h), \underline{\nabla} z)+\left(\underline{A}\left(\underline{\sigma}_{h}\right), \underline{\operatorname{curl}} \Phi\right)+\left(\operatorname{div}\left(\pi_{h}(\underline{\nabla} z)\right), u_{h}\right) .
$$

Now by the first equation of the discrete formulation (1.14):

$$
\left(\underline{A}\left(\underline{\sigma}_{h}\right), \underline{\tau}_{h}\right)+\left(u_{h}, \operatorname{div} \underline{\tau}_{h}\right)=0, \quad \forall \underline{\tau}_{h} \in X_{h} .
$$

Applying (1.73) with $\underline{\tau}_{h}=\pi_{h}(\underline{\nabla} z)$ and $\underline{\tau}_{h}=\underline{\text { curl }}\left(I_{c l}(\Phi)\right)$ successively, we obtain the following equations:

$$
\left(\operatorname{div}\left(\pi_{h}(\underline{\nabla} z)\right), u_{h}\right)=-\left(\underline{A}\left(\underline{\sigma}_{h}\right), \pi_{h}(\underline{\nabla} z)\right)
$$

and

$$
\left(\underline{A}\left(\underline{\sigma}_{h}\right), \underline{\operatorname{curl}}\left(I_{c l}(\Phi)\right)\right)=0 .
$$

Injecting the relations (1.74) and (1.75) in the right-hand side of (1.72), we obtain :

$$
\begin{aligned}
<R, \underline{\tau}>= & \left(\underline{A}(\underline{\sigma} h), \underline{\nabla} z-\pi_{h}(\underline{\nabla} z)\right)+\left(\underline{A}(\underline{\sigma} h), \underline{\operatorname{curl}}\left(\Phi-I_{c l}(\Phi)\right)\right) \\
= & \left(\underline{A}(\underline{\sigma} h), \underline{\nabla} z-\pi_{h}(\underline{\nabla} z)\right)+\sum_{K \in \mathcal{T}_{h}}\left(\underline{A}(\underline{\sigma} h), \underline{\operatorname{curl}}\left(\Phi-I_{c l}(\Phi)\right)\right) \\
= & \left(\underline{A}(\underline{\sigma} h), \underline{\nabla} z-\pi_{h}(\underline{\nabla} z)\right) \\
& +\sum_{K \in \mathcal{T}_{h}}\left\{\left(\operatorname{curl} \underline{\mathrm{A}}(\underline{\sigma} \mathrm{h}), \Phi-\mathrm{I}_{\mathrm{cl}}(\Phi)\right)-<\underline{\mathrm{A}}(\underline{\sigma} \mathrm{h}) \cdot \underline{\mathrm{t}}, \Phi-\mathrm{I}_{\mathrm{cl}}(\Phi)>_{\partial \mathrm{K}}\right\} \\
& (\text { by Green's formula by adapting }(1.31) \text { p. } 19 \text { of }[10]) \\
= & \sum_{K \in \mathcal{T}_{h}}\left(\underline{A}(\underline{\sigma} h), \underline{\nabla} z-\pi_{h}(\underline{\nabla} z)\right)+\sum_{K \in \mathcal{T}_{h}}\left(\operatorname{curl} \underline{\mathrm{A}}\left(\underline{\sigma}_{\mathrm{h}}\right), \Phi-\mathrm{I}_{\mathrm{cl}}(\Phi)\right) \\
- & \sum_{E \in \mathcal{E}_{h}}<\left[\left[\underline{A}\left(\underline{\sigma}_{h}\right) \cdot \underline{\underline{\theta}}\right]\right]_{E}, \Phi-I_{c l}(\Phi)>_{E} .
\end{aligned}
$$

Remark 1.2. The summation $\sum_{E \in \mathcal{E}_{h}}<\llbracket\left[\underline{A}\left(\underline{\sigma}_{h}\right) \cdot \underline{t} \underline{]}\right]_{E}, \Phi-I_{c l}(\Phi)>_{E}$ appearing in the right-hand side of formula $(1.76)$ contains the terms $\left.<\llbracket \underline{A}\left(\underline{\sigma}_{h}\right) \cdot \underline{t}\right]_{E}, \Phi-$ $I_{c l}(\Phi)>_{E}$ where $E$ is an edge of the triangulation $\mathcal{T}_{h}$ contained in the boundary of $\Omega$; in this case it must be understood that $\underline{A}\left(\underline{\sigma}_{h}\right) \cdot \underline{t}$ is 0 outside of $\Omega$. Note also that $[[\underline{A}(\underline{\sigma}) \cdot \underline{t}]]_{E}=0$ for every $E \in \mathcal{E}_{h}$ contained in $\partial \Omega$.

We now define the error estimator $\eta_{1}$ :

Definition 1.1. For each $K \in \mathcal{T}_{h}$, we define $\eta_{1}(K)$ by:

$$
\begin{aligned}
\eta_{1}(K)^{p} & =h_{K}^{p}\left\|\underline{A}\left(\underline{\sigma}_{h}\right)\right\|_{0, p, K}^{p}+h_{K}^{p}\left\|\operatorname{curl}\left(\underline{\mathrm{A}}\left(\underline{\sigma}_{\mathrm{h}}\right)\right)\right\|_{0, \mathrm{p}, \mathrm{K}}^{\mathrm{p}} \\
& \left.+\sum_{E \subset \partial K} h_{E} \| \llbracket\left[\underline{A}\left(\underline{\sigma}_{h}\right) \cdot \underline{t}\right]\right]_{E} \|_{0, p, E}^{p}
\end{aligned}
$$

and we define $\eta_{1}$ by

$$
\eta_{1}=\left[\sum_{K \in \mathcal{T}_{h}} \eta_{1}(K)^{p}\right]^{1 / p}
$$


We are now in a position to bound $\|R\|$ by a constant times the error estimator $\eta_{1}$

\section{Theorem 1.2.}

$$
\|R\| \lesssim \eta_{1}
$$

Proof. It follows from lemma 1.5 that for every $\underline{\tau} \in X$ :

$$
\begin{aligned}
&|<R, \underline{\tau}>| \lesssim \sum_{K \in \mathcal{T}_{h}}\left\|\underline{A}\left(\underline{\sigma}_{h}\right)\right\|_{0, p, K}\left\|\underline{\nabla} z-\pi_{h} \underline{\nabla} z\right\|_{0, q, K} \\
&+\sum_{K \in \mathcal{T}_{h}}\left\|\operatorname{curl} \underline{\mathrm{A}}\left(\underline{\sigma}_{\mathrm{h}}\right)\right\|_{0, \mathrm{p}, \mathrm{K}}\left\|\Phi-\mathrm{I}_{\mathrm{cl}}(\Phi)\right\|_{0, \mathrm{q}, \mathrm{K}} \\
&+\sum_{E \in \mathcal{E}_{h}}\left\|\left[\left[\underline{A}\left(\underline{\sigma}_{h}\right) \cdot \underline{t}\right]\right]_{E}\right\|_{0, p, E}\left\|\Phi-I_{c l}(\Phi)\right\|_{0, q, E} .
\end{aligned}
$$

By (3.6) p. 72 of [1] (see also lemma 3.1 p. 125 of [6]):

$$
\| \underline{\nabla} z-\left.\pi_{h} \underline{\nabla} z\right|_{0, q, K} \lesssim h_{K}|\underline{\nabla} z|_{1, q, K}
$$

By lemma 3.1 p. 57 of [12]:

$$
\| \Phi-\left.I_{c l}(\Phi)\right|_{0, q, K} \lesssim h_{K}|\Phi|_{1, q, \omega_{K}}
$$

and

$$
\| \Phi-\left.I_{c l}(\Phi)\right|_{0, q, E} \lesssim h_{E}^{1 / p}|\Phi|_{1, q, \omega_{E}} .
$$

Let us recall that $\omega_{K}$ denotes the union of $K$ with all the triangles from the triangulation $\mathcal{T}_{h}$ adjacent to the triangle $K$ and that $\omega_{E}$ denotes the union of the at most two triangles of $\mathcal{T}_{h}$ admitting $E$ as an edge. Using inequalities (1.79)-(1.81) to bound the right-hand side of inequality (1.78), we obtain:

$$
\begin{aligned}
& |<R, \underline{\tau}>| \lesssim \sum_{K \in \mathcal{T}_{h}} h_{K}\left|\underline{A}\left(\underline{\sigma}_{h}\right)\right|_{0, p, K}|\underline{\nabla} z|_{1, q, K} \\
& +\sum_{K \in \mathcal{T}_{h}} h_{K}|| \operatorname{curl} \underline{\mathrm{A}}\left(\underline{\sigma}_{\mathrm{h}}\right)||_{0, \mathrm{p}, \mathrm{K}}|\Phi|_{1, \mathrm{q}, \omega_{\mathrm{K}}} \\
& +\sum_{E \in \mathcal{E}_{h}} h_{E}^{1 / p}|| \llbracket\left[\underline{A}\left(\underline{\sigma}_{h}\right) \cdot \underline{t}\right]_{E}||_{0, p, E}|\Phi|_{1, q, \omega_{E}} \\
& \lesssim\left(\sum_{K \in \mathcal{T}_{h}} h_{K}^{p}\left\|\underline{A}\left(\underline{\sigma}_{h}\right)\right\|_{0, p, K}^{p}\right)^{1 / p}|\underline{\nabla} z|_{1, q, \Omega} \\
& +\left(\sum_{K \in \mathcal{T}_{h}} h_{K}^{p}\left\|\operatorname{curl} \underline{\mathrm{A}}\left(\underline{\sigma}_{\mathrm{h}}\right)\right\|_{0, \mathrm{p}, \mathrm{K}}^{\mathrm{p}}\right)^{1 / p}|\Phi|_{1, q, \Omega} \\
& +\left(\sum_{E \in \mathcal{E}_{h}} h_{E}\left\|[[\underline{A}(\underline{\sigma} h) \cdot \underline{t}]]_{E}\right\|_{0, p, E}^{p}\right)^{1 / p}|\Phi|_{1, q, \Omega}
\end{aligned}
$$


and so:

$$
\begin{aligned}
|<R, \underline{\tau}>| \lesssim & \left\{\sum _ { K \in \mathcal { T } _ { h } } \left(h_{K}^{p}\left\|\underline{A}\left(\underline{\sigma}_{h}\right)\right\|_{0, p, K}^{p}+h_{K}^{p}\left\|\operatorname{curl} \underline{\mathrm{A}}\left(\underline{\sigma}_{\mathrm{h}}\right)\right\|_{0, \mathrm{p}, \mathrm{K}}^{\mathrm{p}}\right.\right. \\
& \left.\left.+\sum_{E \subset \partial K} h_{E}|| \llbracket \underline{A}\left(\underline{\sigma}_{h}\right) \cdot \underline{t} \rrbracket_{E} \|_{0, p, E}^{p}\right)\right\}^{1 / p}\left\{|\underline{\nabla} z|_{1, q, \Omega}^{q}+|\Phi|_{1, q, \Omega}^{q}\right\}^{1 / q} .
\end{aligned}
$$

Using (1.55) and definition 1.1, we obtain:

$$
\begin{aligned}
|<R, \underline{\tau}>| & \lesssim\left(\sum_{K \in \mathcal{T}_{h}} \eta_{1}(K)^{p}\right)^{1 / p}\left(\|\underline{\tau}\|_{0, q, \Omega}+\|\operatorname{div} \underline{\tau}\|_{0, q, \Omega}\right) \\
& \lesssim \eta_{1}\|\underline{\tau}\|_{X}
\end{aligned}
$$

This proves (1.77).

Remark 1.3. As already said in the introduction:

$$
\|r\|=\left\|f-P_{h}^{0} f\right\|_{0, q, \Omega}=\left(\sum_{K \in \mathcal{T}_{h}} \eta_{2}(K)^{q}\right)^{1 / q},
$$

where

$$
\eta_{2}(K)=\left\|f-P_{h}^{0} f\right\|_{0, q, K}, \quad \forall K \in \mathcal{T}_{h} .
$$

Thus $\|r\|$ is immediately bounded in terms of the data. We may now turn to the hybridization of the discretized mixed formulation (1.14).

\section{Hybridization of the discrete mixed formulation}

The construction of a basis of vectorfields in $X_{h}$ presents the difficulty that for a vectorfield $\underline{\tau}_{h}$ to be in $X_{h}$, its normal component must be continuous at each interface between two adjacent triangles of the triangulation $\mathcal{T}_{h}$. To overcome this difficulty, we introduce the enlarged space

$$
\tilde{X}_{h}=\left\{\tilde{\tau}_{h} \in\left[L^{q}(\Omega)\right]^{2} ; \tilde{\tau}_{h \mid K} \in R T_{0}(K), \forall K \in \mathcal{T}_{h}\right\}
$$

and also the space of Lagrange multipliers

$$
\begin{aligned}
\tilde{M}_{h}=\left\{\mu_{h}: \partial \mathcal{T}_{h} \rightarrow \mathbb{R} ; \mu_{h_{\mid e}} \text { is constant for every edge e of } \mathcal{T}_{h}\right. \\
\text { and } \left.\mu_{h_{\mid e}}=0 \text { if the edge } e \subset \partial \Omega\right\} .
\end{aligned}
$$

In $(2.85), \partial \mathcal{T}_{h}$ denotes the "skeleton" of the triangulation $\mathcal{T}_{h}$, i.e. the union of all the edges of the triangulation $\mathcal{T}_{h}$ and for an edge $e$ of $\mathcal{T}_{h}, \stackrel{\circ}{e}$ denotes its "interior", that 
is to say the edge $e$ without its extremities. We are now in a position to introduce the "hybrid formulation" of (1.14): find $\left(\underline{\tilde{\sigma}}_{h}, \tilde{u}_{h}, \lambda_{h}\right) \in \tilde{X}_{h} \times M_{h} \times \tilde{M}_{h}$ such that:

$$
\left\{\begin{aligned}
\int_{\Omega} \underline{A}\left(\underline{\tilde{\sigma}}_{h}\right) \cdot \underline{\tilde{\tau}}_{h} d x+\sum_{K \in \mathcal{T}_{h}} \int_{K} \tilde{u}_{h} \operatorname{div} \underline{\tilde{\tau}}_{h} d x & \\
-\sum_{K \in \mathcal{T}_{h}} \int_{\partial K} \lambda_{h} \tilde{\tilde{\tau}}_{h} \cdot \underline{n}_{K} d s=0 & \forall \tilde{\tilde{\tau}}_{h} \in \tilde{X}_{h}, \\
\sum_{K \in \mathcal{T}_{h}} \int_{K}\left(\operatorname{div} \underline{\tilde{\sigma}}_{h}\right) v_{h} d x+\int_{\Omega} f v_{h} d x=0 & \forall v_{h} \in M_{h}, \\
\sum_{K \in \mathcal{T}_{h}} \int_{\partial K} \mu_{h} \underline{\tilde{\sigma}}_{h} \cdot \underline{n}_{K} d s=0 & \forall \mu_{h} \in \tilde{M}_{h} .
\end{aligned}\right.
$$

In (2.86), $\underline{n}_{K}$ denotes the unit exterior normal to the triangle $K$. Arguing as in [11] p. 527 , it is easily seen that if $\left(\underline{\tilde{\sigma}}_{h}, \tilde{u}_{h}, \lambda_{h}\right) \in \tilde{X}_{h} \times M_{h} \times \tilde{M}_{h}$ is a solution of the hybrid formulation (2.86), then necessarily $\underline{\tilde{\sigma}}_{h}=\underline{\sigma}_{h}$ and $\tilde{u}_{h}=u_{h}$. Now let us prove that (2.86) possesses at most one solution. Thus, let $\left(\underline{\tilde{\sigma}}_{h}, \tilde{u}_{h}, \lambda_{h}\right) \in \tilde{X}_{h} \times M_{h} \times \tilde{M}_{h}$ be a solution of (2.86). We know already that this implies that $\underline{\tilde{\sigma}}_{h}=\underline{\sigma}_{h}$ and $\tilde{u}_{h}=u_{h}$. Let us consider for $\tilde{\tau}_{h} \in \tilde{X}_{h}$ the vectorfield $\tilde{\tau}_{K, e} \in \tilde{X}_{h}$ such that for the edge $e$ of the triangle $K \in \mathcal{T}_{h}, \tilde{\tau}_{K, e} \cdot \underline{n}_{K}$ equals 1 on $e$ and 0 on the two other sides of the triangle $K$, and $\tilde{\tau}_{K, e}$ is null on all other triangles $K^{\prime} \neq K$ of the triangulation $\mathcal{T}_{h}$. Putting it into the first equation of (2.86), we obtain:

$$
|e| \lambda_{h \mid e}=\int_{K} \underline{A}\left(\underline{\sigma}_{h}\right) \cdot \underline{\tilde{\tau}}_{K, e} d x+\int_{K} u_{h} \operatorname{div} \underline{\tilde{\tau}}_{K, e} d x .
$$

Equation (2.87) shows that the Lagrange multiplier $\lambda_{h}$ is also completely determined. Note that the existence of the above vectorfield $\tilde{\tau}_{h}$ is proved in lemma 2.2 of [11]. Thinking a bit to equation (2.87) shows that it proves also the existence of a solution to the nonlinear system (2.86). Taking $\underline{\tilde{\sigma}}_{h}=\underline{\sigma}_{h}$ and $\tilde{u}_{h}=u_{h}$, we can define $\lambda_{h}$ by equation (2.87) noting that these special vectorfields $\tilde{\tau}_{K, e}$ form a basis of the space $\tilde{X}_{h}$. Let us observe that if $e$ has two adjacent triangles $K_{1}$ and $K_{2}$, we may choose any in the right-hand side of equation (2.87) as it gives the same result. This follows from equation (1.14)(i) with $\underline{\underline{\tau}}_{h}$ equals to $\tilde{\tau}_{K_{1}, e}$ on $K_{1},-\tilde{\tau}_{K_{2}, e}$ on $K_{2}$ and 0 on the other triangles of $\mathcal{T}_{h}$. Note also that if the edge $e$ is contained in the boundary of $\Omega$, then $\tilde{\underline{\tau}}_{K, e} \in X_{h}$ and thus by the first equation of (1.14), the right hand side of $(2.87)$ is zero showing that $\lambda_{h \mid e}=0$ in this case. We have thus established the following result:

Proposition 2.1. The hybrid formulation (2.86) possesses one and only one solution $\left(\underline{\tilde{\sigma}}_{h}, \tilde{u}_{h}, \lambda_{h}\right) \in \tilde{X}_{h} \times M_{h} \times \tilde{M}_{h}$. Moreover, $\underline{\tilde{\sigma}}_{h}=\underline{\sigma}_{h}$ and $\tilde{u}_{h}=u_{h}$.

Numerically, we may try to solve the nonlinear problem (2.86) by a simple Picard scheme also called the method of successive substitutions. Thus we construct a sequence $\left(\underline{\sigma}^{m}, u^{m}, \lambda^{m}\right) \in \tilde{X}_{h} \times M_{h} \times \tilde{M}_{h}$ by the following iterative algorithm: Given 
$\left(\underline{\sigma}^{m}, u^{m}, \lambda^{m}\right) \in \tilde{X}_{h} \times M_{h} \times \tilde{M}_{h}$, compute $\left(\underline{\sigma}^{m+1}, u^{m+1}, \lambda^{m+1}\right) \in \tilde{X}_{h} \times M_{h} \times \tilde{M}_{h}$ solution of the linear system

$$
\left\{\begin{aligned}
\int_{\Omega}\left|\underline{\sigma}^{m}\right|^{q-2} \underline{\sigma}^{m+1} \cdot \tilde{\tau}_{h} d x+\sum_{K \in \mathcal{T}_{h}} \int_{K} u^{m+1} \operatorname{div} \tilde{\tilde{\tau}}_{h} d x & \\
-\sum_{K \in \mathcal{T}_{h}} \int_{\partial K} \lambda^{m+1} \underline{\tilde{\tau}}_{h} \cdot \underline{n}_{K} d s=0 & \forall \tilde{\tilde{\tau}}_{h} \in \tilde{X}_{h}, \\
\sum_{K \in \mathcal{T}_{h}} \int_{K}\left(\operatorname{div} \underline{\sigma}^{m+1}\right) v_{h} d x+\int_{\Omega} f v_{h} d x=0 & \forall v_{h} \in M_{h}, \\
\sum_{K \in \mathcal{T}_{h}} \int_{\partial K} \mu_{h} \underline{\sigma}^{m+1} \cdot \underline{n}_{K} d s=0 & \forall \mu_{h} \in \tilde{M}_{h} .
\end{aligned}\right.
$$

It is not evident that the linear system (2.88) possesses one and only one solution $\left(\underline{\sigma}^{m+1}, u^{m+1}, \lambda^{m+1}\right) \in \tilde{X}_{h} \times M_{h} \times \tilde{M}_{h}$. Let us observe that if $\left(\underline{\sigma}^{m+1}, u^{m+1}, \lambda^{m+1}\right)$ is a solution to (2.88), then a fortiori $\left(\underline{\sigma}^{m+1}, u^{m+1}\right) \in X_{h} \times M_{h}$ due to the third equation of (2.88) and is solution of the following linear system:

$$
\left\{\begin{aligned}
\int_{\Omega}\left|\underline{\sigma}^{m}\right|^{q-2} \underline{\sigma}^{m+1} \cdot \underline{\tau}_{h} d x+\int_{\Omega} u^{m+1} \operatorname{div} \underline{\tau}_{h} d x=0 & \forall \underline{\tau}_{h} \in X_{h}, \\
\int_{\Omega} \operatorname{div} \underline{\sigma}^{m+1} v_{h} d x+\int_{\Omega} f v_{h} d x=0 & \forall v_{h} \in M_{h} .
\end{aligned}\right.
$$

We first give a sufficient condition for the linear system (2.89) to have one and only one solution.

Proposition 2.2. If for every triangle $K$ of the triangulation $\mathcal{T}_{h}, \underline{\sigma}^{m}|K| \equiv 0$, then the linear system (2.89) possesses one and only one solution $\left(\underline{\sigma}^{m+1}, u^{m+1}\right) \in$ $X_{h} \times M_{h}$.

Proof. By corollary I.4.1 of [8] it suffices to prove that the bilinear form

$$
a(., .): X_{h} \times X_{h} \rightarrow \mathbb{R}:\left(\underline{\tau}_{h}^{\prime}, \underline{\tau}_{h}^{\prime \prime}\right) \mapsto \int_{\Omega}\left|\underline{\sigma}^{m}\right|^{q-2} \underline{\tau}_{h}^{\prime} \cdot \underline{\tau}_{h}^{\prime \prime} d x
$$

is coercive on $V_{h}=\left\{\underline{\tau}_{h} \in X_{h} ; \int_{\Omega} \operatorname{div} \underline{\tau}_{h} v_{h} d x=0, \forall v_{h} \in M_{h}\right\}$. One must in fact check also that the bilinear form

$$
b(., .): X_{h} \times M_{h} \rightarrow \mathbb{R}:\left(\underline{\tau}_{h}, v_{h}\right) \mapsto \int_{\Omega} \operatorname{div} \underline{\tau}_{h} v_{h} d x
$$

satisfies the inf-sup condition but this results from proposition 3.1 of [1]. Let us consider $\underline{\tau}_{h} \in V_{h}$. As $\underline{\tau}_{h \mid K} \in R T_{0}(K), \forall K \in \mathcal{T}_{h}$, it follows immediately from the condition $\underline{\tau}_{h} \in V_{h}$ that $\underline{\tau}_{h}$ must be constant on each triangle $K$ of the triangulation $\mathcal{T}_{h}$. Thus for such a vectorfield, one has:

$$
a\left(\underline{\tau}_{h}, \underline{\tau}_{h}\right)=\int_{\Omega}\left|\underline{\sigma}^{m}\right|^{q-2}\left|\underline{\tau}_{h}\right|^{2} d x=\sum_{K \in \mathcal{T}_{h}}\left|\underline{\tau}_{h \mid K}\right|^{2} \int_{K}\left|\underline{\sigma}^{m}\right|^{q-2} d x>0 .
$$


Due to our hypothesis on $\underline{\sigma}^{m}$, it follows that:

$$
c=\inf _{K \in \mathcal{T}_{h}} \frac{1}{|K|} \int_{K}\left|\underline{\sigma}^{m}\right|^{q-2} d x>0 .
$$

Thus:

$$
a\left(\underline{\tau}_{h}, \underline{\tau}_{h}\right) \geq c\left[\left(\sum_{K \in \mathcal{T}_{h}}|K|\left|\underline{\tau}_{h \mid K}\right|^{2}\right)^{1 / 2}\right]^{2} \sim\left\|\underline{\tau}_{h}\right\|^{2},
$$

all norms being equivalent on finite dimensional vector spaces. This proves the coercivity of the bilinear form $a(.,$.$) on V_{h}$.

Now, it remains to prove that under the same condition on $\underline{\sigma}^{m}$, that the linear $\operatorname{system}(2.88)$ possesses one and only one solution $\left(\underline{\sigma}^{m+1}, u^{m+1}, \lambda^{m+1}\right) \in \tilde{X}_{h} \times M_{h} \times$ $\tilde{M}_{h}$. This is the purpose of the following corollary.

Corollary 2.1. If for every triangle $K$ of the triangulation $\mathcal{T}_{h}, \underline{\sigma}_{\mid K}^{m} \not \equiv 0$, then the linear system (2.88) possesses one and only one solution $\left(\underline{\sigma}^{m+1}, u^{m+1}, \lambda^{m+1}\right) \in$ $\tilde{X}_{h} \times M_{h} \times \tilde{M}_{h}$.

Proof. We know already that the linear system (2.89) possesses one and only one solution $\left(\underline{\sigma}^{m+1}, u^{m+1}\right) \in X_{h} \times M_{h}$. Now, we put that solution in system (2.88) and define $\lambda^{m+1}$ by the first equation of (2.88). More precisely, we define $\lambda_{\mid e}^{m+1}$ by:

$$
|e| \lambda^{m+1}{ }_{\mid e}=\int_{K}\left|\underline{\sigma}^{m}\right|^{q-2} \underline{\sigma}^{m+1} \cdot \underline{\tilde{\tau}}_{K, e} d x+\int_{K} u^{m+1} \operatorname{div} \underline{\tilde{\tau}}_{K, e} d x,
$$

where $K$ is an arbitrary adjacent triangle to the edge $e$ and $\tilde{\tau}_{K, e}$ is the same vectorfield that appears in formula (2.87). Let us note that if the edge $e$ is contained in the boundary of $\Omega$, then $\tilde{\tau}_{K, e} \in X_{h}$ and thus the first equation of (2.89) implies that $\lambda_{\mid e}^{m+1}$ defined by (2.90) must be 0 . Thus $\lambda^{m+1} \in \tilde{M}_{h}$ which proves that the linear system (2.88) possesses one and only one solution.

Definition 2.1. In the sequel, we will denote for a triangle $K \in \mathcal{T}_{h}$ by $\underline{x}_{K}^{m}$ the barycenter of the probability measure of density $\frac{\left.\left|\underline{\sigma}^{m}\right| K\right|^{q-2}}{\int_{K}\left|\underline{\sigma}^{m}{ }^{m}\right|^{q-2} d x}$ with respect to Lebesgue measure on $\mathbb{R}^{2}$ restricted to $K$ that is ([13] p. 146):

$$
\underline{x}_{K}^{m}=\frac{1}{\int_{K}\left|\underline{\sigma}_{\mid K}^{m}\right|^{q-2} d x} \int_{K} \underline{x}\left|\underline{\sigma}^{m}(\underline{x})\right|^{q-2} d x .
$$

It is well defined for every $K \in \mathcal{T}_{h}$ under the hypothesis of proposition 2.2 on $\underline{\sigma}^{m}$.

Proposition 2.3. Under the hypothesis of proposition 2.2 on $\underline{\sigma}^{m}$, for every triangle $K$ of the triangulation $\mathcal{T}_{h}$ we have:

$$
\left(\underline{\sigma}^{m+1}{ }_{\mid K}\right)(\underline{x})=\frac{\int_{\partial K} \lambda^{m+1} \underline{n}_{K} d s}{\int_{K}\left|\underline{\sigma}^{m}\right|^{q-2} d x}-\frac{\int_{K} f d x}{2|K|}\left(\underline{x}-\underline{x}_{K}^{m}\right) \quad \forall x \in K
$$


where $|K|$ denotes the area of the triangle $K$.

Proof. Let $K$ be an arbitrary triangle of the triangulation $\mathcal{T}_{h}$. Putting in the first equation of (2.88), any vector field $\tilde{\tau}_{h}$ constant on $K$ and nul outside $K$, we obtain :

$$
\int_{K}\left|\underline{\sigma}_{\mid K}^{m}\right|^{q-2} \underline{\sigma}_{\mid K}^{m+1} d x=\int_{\partial K} \lambda^{m+1} \underline{n}_{K} d s .
$$

Writing the unknown vectorfield $\underline{\sigma}^{m+1}{ }_{\mid K}$ in the form:

$$
\underline{\sigma}_{\mid K}^{m+1}(\underline{x})=\underline{a}+c\left(\underline{x}-\underline{x}_{K}^{m}\right)
$$

where $\underline{a} \in \mathbb{R}^{2}, c \in \mathbb{R}$ and $\underline{x}_{K}^{m}$ has been defined in definition (2.1), it follows from equation (2.92):

$$
\underline{a} \int_{K}\left|\underline{\sigma}_{\mid K}^{m}\right|^{q-2} d x=\int_{\partial K} \lambda^{m+1} \underline{n}_{K} d s .
$$

Thus we have:

$$
\underline{a}=\frac{\int_{\partial K} \lambda^{m+1} \underline{n}_{K} d s}{\left.\int_{K}\left|\underline{\sigma}^{m}\right|_{\mid K}\right|^{q-2} d x} .
$$

By the second equation of (2.88):

$$
\operatorname{div} \underline{\sigma}_{\mid K}^{m+1}=-\frac{1}{|K|} \int_{K} f d x .
$$

By (2.93), we have also

$$
\operatorname{div} \underline{\sigma}_{\mid K}^{m+1}=2 c
$$

From (2.93), (2.95), (2.96) and (2.97) follows our claim (2.91).

For $u_{\mid K}^{m+1}$, we have the following formula:

Proposition 2.4. Under the hypothesis of proposition 2.2 on $\underline{\sigma}^{m}$, for every triangle $K$ of the triangulation $\mathcal{T}_{h}$ we have:

$$
\begin{aligned}
u_{\mid K}^{m+1} & =\left(\int_{K}\left|\underline{\sigma}_{\mid K}^{m}\right|^{q-2}\left|\underline{x}-\underline{x}_{K}^{m}\right|^{2} d x\right) \frac{\int_{K} f d x}{4|K|^{2}} \\
& +\frac{1}{2|K|} \int_{\partial K} \lambda^{m+1}\left(\underline{x}-\underline{x}_{K}^{m}\right) \cdot \underline{n}_{K} d s .
\end{aligned}
$$

Proof. Let us take for the vectorfield $\tilde{\tau}_{h}$ in the first equation of (2.88), the vectorfield equals to $\underline{x}-\underline{x}_{K}^{m}$ in the triangle $K$ and to 0 outside of $K$. We obtain using (2.91) and the fact that $\int_{K}\left|\underline{\sigma}^{m}(\underline{x})\right|^{q-2}\left(\underline{x}-\underline{x}_{K}^{m}\right) d x=0$ :

$$
\begin{aligned}
-\int_{K}\left|\underline{\sigma}^{m}\right|^{q-2}(\underline{x})\left|\underline{x}-\underline{x}_{K}^{m}\right|^{2} d x & \cdot \frac{1}{2|K|} \int_{K} f d x+2|K| u_{\mid K}^{m+1} \\
& =\int_{\partial K} \lambda^{m+1}\left(\underline{x}-\underline{x}_{K}^{m}\right) \cdot \underline{n}_{K} d s .
\end{aligned}
$$


From this last equation, (2.98) follows easily.

Remark 2.1. Equations (2.91) and (2.98) show that $\underline{\sigma}^{m+1}{ }_{\mid K}$ and $u_{\mid K}^{m+1}$ are completely defined by the restriction of the Lagrange multiplier $\lambda^{m+1}$ to the boundary of $K$. Thus, we are reduced to the determination of the Lagrange multiplier $\lambda^{m+1}$. To obtain the linear system from which $\lambda^{m+1}$ is the solution, it suffices to express that $\underline{\sigma}^{m+1}$ has its normal component continuous across each interior edge $e$ of the triangulation $\mathcal{T}_{h}$

Proposition 2.5. The Lagrange multiplier $\lambda^{m+1}$ is the solution of the linear system obtained by associating to each interior edge e of the triangulation the equation:

$$
\begin{aligned}
& \frac{1}{\int_{K_{1}}\left|\underline{\sigma}_{K_{1}}^{m}\right|^{q-2} d x}\left[|e| \lambda_{\mid e}^{m+1}-\frac{\left|d_{1}\right|^{2}+|e|^{2}-\left|g_{1}\right|^{2}}{2|e|} \lambda_{\mid d_{1}}^{m+1}-\frac{\left|g_{1}\right|^{2}+|e|^{2}-\left|d_{1}\right|^{2}}{2|e|} \lambda_{\mid g_{1}}^{m+1}\right] \\
+ & \frac{1}{\int_{K_{2}}\left|\underline{\sigma}_{K_{2}}^{m}\right|^{q-2} d x}\left[|e| \lambda_{\mid e}^{m+1}-\frac{\left|d_{2}\right|^{2}+|e|^{2}-\left|g_{2}\right|^{2}}{2|e|} \lambda_{\mid d_{2}}^{m+1}-\frac{\left|g_{2}\right|^{2}+|e|^{2}-\left|d_{2}\right|^{2}}{2|e|} \lambda_{\mid g_{2}}^{m+1}\right] \\
= & \left(\frac{1}{2\left|K_{1}\right|} \int_{K_{1}} f(y) d y\right)\left(\underline{x}-\underline{x}_{K_{1}}^{m}\right) \cdot \underline{n}_{K_{1} \mid e}+\left(\frac{1}{2\left|K_{2}\right|} \int_{K_{2}} f(y) d y\right)\left(\underline{x}-\underline{x}_{K_{2}}^{m}\right) \cdot \underline{n}_{K_{2} \mid e}
\end{aligned}
$$

In equation (2.99), $K_{1}$ and $K_{2}$ denote the two triangles of $\mathcal{T}_{h}$ sharing in common the edge $e . d_{1}$ and $g_{1}$ (resp. $d_{2}$ and $g_{2}$ ) denote the two other sides than $e$ of the triangle $K_{1}$ (resp. $K_{2}$ ) (for an arbitrary edge $b$ of $\mathcal{T}_{h},|b|$ denotes its length). In the right-hand side of equation (2.99), $\underline{x}$ denotes an arbitrary point of the edge $e$ (usually we will take its midpoint).

Proof. Before beginning really the proof, let us explain why $\underline{x}$ is an arbitrary point of $e$ in the right-hand side of (2.99). Let us take two arbitrary points $\underline{x}^{\prime}$ and $\underline{x}^{\prime \prime}$ on e. Then

$$
\left(\underline{x}^{\prime}-\underline{x}_{K_{i}}^{m}\right) \cdot \underline{n}_{K_{i} \mid e}=\left(\underline{x}^{\prime \prime}-\underline{x}_{K_{i}}^{m}\right) \cdot \underline{n}_{K_{i} \mid e}
$$

for $i=1,2$ as $\underline{x}^{\prime}-\underline{x}^{\prime}$ is orthogonal to $\underline{n}_{K_{i} \mid e}$. Now let us prove (2.99). To do that, let us express that

$$
\underline{\sigma}_{\mid K_{1}}^{m+1} \cdot \underline{n}_{K_{1} \mid e}+\underline{\sigma}_{\mid K_{2}}^{m+1} \cdot \underline{n}_{K_{2} \mid e}=0
$$

using formula (2.91). To alleviate the notations, we will write in the sequel $\underline{n}_{K_{i}}^{e}$ for $\underline{n}_{K_{i} \mid e},(i=1,2)$. We obtain from $(2.91)$ :

$$
\begin{aligned}
& \frac{\int_{\partial K_{1}} \lambda^{m+1} \underline{n}_{K_{1}} \cdot \underline{n}_{K_{1}}^{e} d s}{\int_{K_{1}}\left|\underline{\sigma}^{m}\right|^{q-2} d x}+\frac{\int_{\partial K_{2}} \lambda^{m+1} \underline{n} K_{2} \cdot \underline{n}_{K_{2}}^{e} d s}{\int_{K_{2}}\left|\underline{\sigma}^{m}\right|^{q-2} d x} \\
= & \frac{\int_{K_{1}} f d x}{2\left|K_{1}\right|}\left(\underline{x}-\underline{x}_{K_{1}}^{m}\right) \cdot \underline{n}_{K_{1}}^{e}+\frac{\int_{K_{2}} f d x}{2\left|K_{2}\right|}\left(\underline{x}-\underline{x}_{K_{2}}^{m}\right) \cdot \underline{n}_{K_{2}}^{e}
\end{aligned}
$$


for every $\underline{x} \in e$; but due to (2.100) it suffices to express it for one $\underline{x} \in e$. Decomposing each of the integral above on $\partial K_{i} \quad(i=1,2)$ as sums of integrals on each side, we obtain:

$$
\begin{aligned}
& \frac{1}{\int_{K_{1}}\left|\underline{\sigma}^{m}\right|^{q-2} d x}\left[|e| \lambda_{\mid e}^{m+1}-\left|d_{1}\right| \cos \alpha_{1} \lambda_{\mid d_{1}}^{m+1}-\left|g_{1}\right| \cos \beta_{1} \lambda_{\mid g_{1}}^{m+1}\right] \\
+ & \frac{1}{\int_{K_{2}}\left|\underline{\sigma}^{m}\right|^{q-2} d x}\left[|e| \lambda_{\mid e}^{m+1}-\left|d_{2}\right| \cos \alpha_{2} \lambda_{\mid d_{2}}^{m+1}-\left|g_{2}\right| \cos \beta_{2} \lambda_{\mid g_{2}}^{m+1}\right] \\
= & \frac{\int_{K_{1}} f d x}{2\left|K_{1}\right|}\left(\underline{x}-\underline{x}_{K_{1}}^{m}\right) \cdot \underline{n}_{K_{1}}^{e}+\frac{\int_{K_{2}} f d x}{2\left|K_{2}\right|}\left(\underline{x}-\underline{x}_{K_{2}}^{m}\right) \cdot \underline{n}_{K_{2}}^{e},
\end{aligned}
$$

where $\alpha_{i}$ (resp. $\beta_{i}$ ) denotes the angle in the triangle $K_{i}$ between the sides $d_{i}$ and $e$ (resp. $g_{i}$ and $\left.e\right),(i=1,2)$. Using the law of cosines we have:

$$
\cos \alpha_{i}=\frac{\left|d_{i}\right|^{2}+|e|^{2}-\left|g_{i}\right|^{2}}{2\left|d_{i}\right||e|} \text { and } \cos \beta_{i}=\frac{\left|g_{i}\right|^{2}+|e|^{2}-\left|d_{i}\right|^{2}}{2\left|g_{i}\right||e|},
$$

and replacing in (2.102) we obtain (2.99).

\section{Numerical Experiments}

The following experiments will underline and confirm our theoretial predictions. These numerical tests consists in solving the mixed formulation of the $p$-Laplace equation (0.1) for the value of $f$ given by $(0.7)$ with $\varepsilon=0.05$ on the unit square $\Omega=$ ]$-1 / 2,1 / 2\left[^{2}\right.$, using the Raviart-Thomas finite element of degree 0 . The correponding exact solution is given by (0.6) and displayed on Figure 1.

The computations are performed for $p=1.8$ and $p=3.0$, each of these two values ensuring that $f \in L^{q}(\Omega)$, with $\frac{1}{p}+\frac{1}{q}=1$. Firstly, with the knowledge of the exact solution $u$ given by (0.6), the order of convergence (function of $p$ ) is verified, using theorem 3.2 of [1] for $p=1.8$ and theorem 3.1 of [1] for $p=3.0$ (one verifies that $(\underline{\sigma}, u) \in W^{1, q}(\Omega)^{2} \times W^{1, p}(\Omega)$ for $p=1.8$ or $\left.p=3.0\right)$. Secondly, the ratios between the errors and the estimators are plotted, to verify the reliability of the estimators. The numerical tests are performed using quasi-uniform meshes similar to the one displayed on Figure 2, and characterized by the number of nodes $n$ in each direction of space. If we note $h$ the diameter of any triangle and DoF the number of degree of freedom associated to the mesh (i.e. the number of segments), we have $h=\sqrt{2} /(n-1)$ and $D o F=(n-1)(3 n-1)$.

\subsection{Order of convergence of the methods}

Let us quantify how the numerical solution converges towards the exact one. To this end, we plot the curves :

- $\left\|\underline{\sigma}_{h}-\underline{\sigma}\right\|_{0, q, \Omega}$ as a function of $D o F$ (see Fig. 3),

- $\left\|u-u_{h}\right\|_{0, p, \Omega}$ as a function of DoF (see Fig. 4). 
The predicted orders of convergence in $h$ are given in [1], and summarized in table 1. The corresponding order of convergence in DoF are mentionned into brackets $\left(D o F=O\left(h^{-2}\right)\right)$.

\begin{tabular}{|c|c|c|}
\hline & $\left\|\underline{\sigma}_{h}-\underline{\sigma}\right\|_{0, q, \Omega}$ & $\left|u-u_{h}\right|_{0, p, \Omega}$ \\
\hline$p=1.8$ & $\left(-\frac{4}{9}\right)$ & $\left.\frac{1}{2}\right)$ \\
\hline$p=3$ & $\frac{3}{4} \quad\left(-\frac{3}{8}\right)$ & $\left(-\frac{1}{4}\right)$ \\
\hline
\end{tabular}

Table 1. The predicted orders of convergence in $h$ (and in $D o F)$.

For $p=1.8$, the numerical results always give slightly better orders of convergence asymptotically whatever the error considered. For $p=3.0$, the good order of convergence is asymptotically achieved for the error on $\sigma_{h}$, and a better order of convergence than the one expected by the theory is obtained for the error on $u_{h}$.

\subsection{Reliability of the estimators}

Proposition 1.5 proves that $\|r\|=\eta_{2}$, and theorem 1.2 allows to bound $\|R\|$ by a constant times the error estimator $\eta_{1}$. As a consequence, following proposition 1.3 (resp. proposition 1.4), we plot on figure 5 the ratio $r_{1}$ (resp. $r_{2}$ ) defined for $p=1.8$ by :

$$
\left\{\begin{array}{l}
r_{1}=\frac{\|\underline{\sigma} h-\underline{\sigma}\|_{0, q, \Omega}}{\eta_{1}^{p / q}+\eta_{2}^{2 / q}+\left(\eta_{2} \eta_{1}\right)^{1 / q}}, \\
r_{2}=\frac{\left\|u-u_{h}\right\|_{0, p, \Omega}}{\eta_{1}+\eta_{2}+\eta_{1}^{p / 2}}
\end{array}\right.
$$

Similarly, following proposition 1.2 (resp. corollary 1.1), we plot on figure 6 the ratio $r_{3}$ (resp. $r_{4}$ ) defined for $p=3.0$ by :

$$
\left\{\begin{array}{l}
r_{3}=\frac{\left\|\underline{\sigma}_{h}-\underline{\sigma}\right\|_{0, q, \Omega}}{\sqrt{\eta_{1}^{2}+\eta_{2}^{q}+\eta_{2} \eta_{1}}}, \\
r_{4}=\frac{\left\|u-u_{h}\right\|_{0, p, \Omega}}{\eta_{1}+\left(\eta_{1}^{2}+\eta_{2}^{q}+\eta_{2} \eta_{1}\right)^{1 / p}} .
\end{array}\right.
$$

The meshes used are the same than the ones involved in part (3.1). We see that whatever the ratio $r_{i}$ chosen $(1 \leq i \leq 4)$, the ratio is asymptotically constant. In other words, it appears that all these ratios are asymptotically independent of the number of degrees of freedom DoF associated to the mesh. As a consequence, it shows that the estimators are actually reliable. 
Remark 3.1. Our computations show that the effectivity index, which is equal to $1 / r_{i}, 1 \leq i \leq 4$, is very high for some of the examples. This fact is due to the presence of unknown constants that enter into the calculation. Using recovery type estimators, one can adjust these constants properly to match the predictions of the residual and recovery estimators.

Remark 3.2. Computations have been performed with the use of the code Simula+ developed by the LAMAV laboratory (University of Valenciennes, France) and the LPMM laboratory (ENSAM of Metz, France).

\section{References}

1. M. Farhloul, H. Manouzi, On a mixed finite element method for the $p$-Laplacian, Canadian Applied Mathematics Quarterly 8 (2000) 67-78.

2. J.M. Thomas, Sur l'analyse numérique des méthodes d'éléments finis hybrides et mixtes, Thèse de l'université Pierre et Marie Curie, Paris, 1977.

3. P.A. Raviart, J.M. Thomas, A mixed finite element method for second order elliptic problems, Mathematical aspects of finite element methods, edited by I. Galligani and E. Magenes, Lecture Notes in Mathematics 606, Springer-Verlag, 292-315, 1977.

4. P.G. Ciarlet, The Finite Element Method for Elliptic Problems, North-Holland, Amsterdam, 1978.

5. D. Sandri, Sur l'approximation numérique des écoulements quasi-Newtoniens dont la viscosité suit la loi puissance ou la loi de Carreau, M² AN 27 (1993) 131-155.

6. M. Farhloul, A mixed finite element method for a nonlinear Dirichlet problem, IMA Journal of Numerical Analysis 18 (1998) 121-132.

7. P. Grisvard, Elliptic Problems in Nonsmooth Domains, Monographs and Studies in Mathematics 24, Pitman, 1985.

8. V. Girault, P.A. Raviart, Finite Element Methods for Navier-Stokes Equations. Theory and algorithms, SCM5, Springer-Verlag, 1986.

9. R. Dautray, J.-L. Lions, Analyse mathématique et calcul numérique pour les sciences et les techniques, volume 3 : Transformations, Sobolev, Opérateurs. CEA, INSTN, Collection Enseignement, Masson, 1988.

10. Z. Mghazli, Une méthode mixte pour les problèmes d'hydrodynamique, Thèse de l'université de Montréal, 1987.

11. H. El Sossa, L. Paquet, Refined mixed finite element method for the Poisson problem on a polygonal domain with a reentrant corner, Advances in Mathematical Sciences and Applications, Gakkotosho, Tokyo 12 (2002) 607-645.

12. R. Verfürth, A Review of a posteriori Error Estimation and Adaptive MeshRefinement Techniques. Wiley and Teubner, 1996.

13. J. Dieudonné, Eléments d'Analyse, tome II. Gauthier-Villars (Paris), Cahiers Scientifiques, Fascicule XXXI, 1969. 


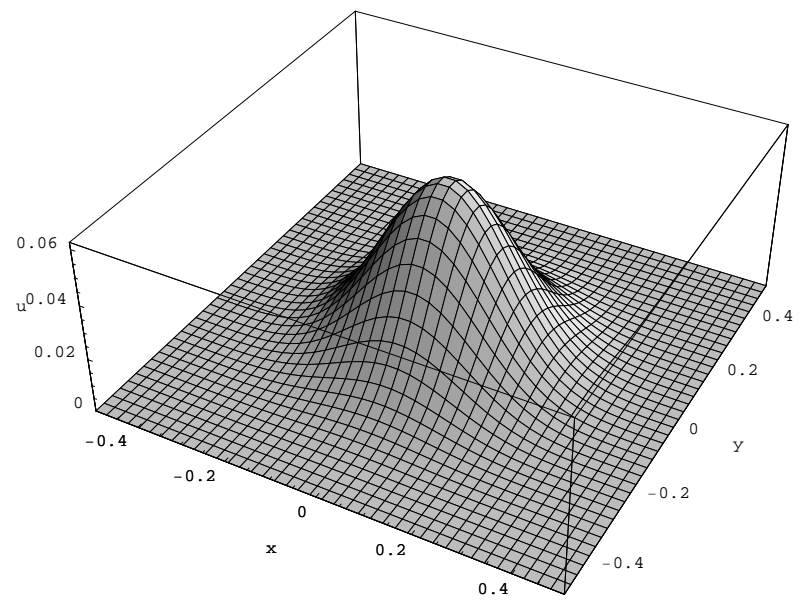

Fig. 1. The exact solution $u$.

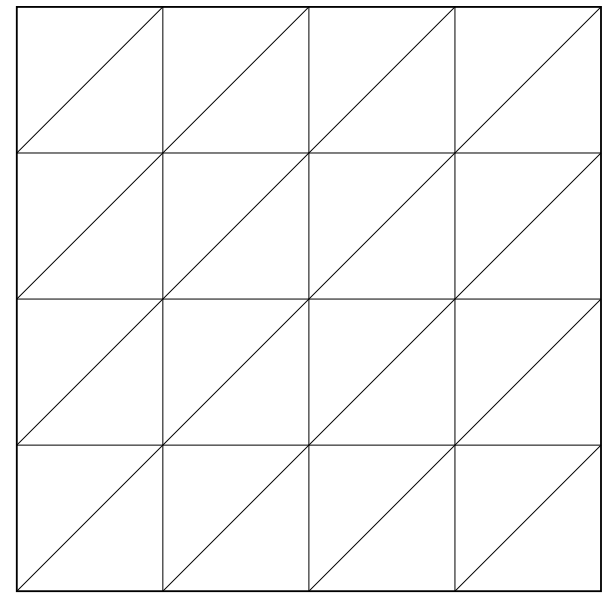

Fig. 2. Quasi-uniform mesh with $n=5$. 

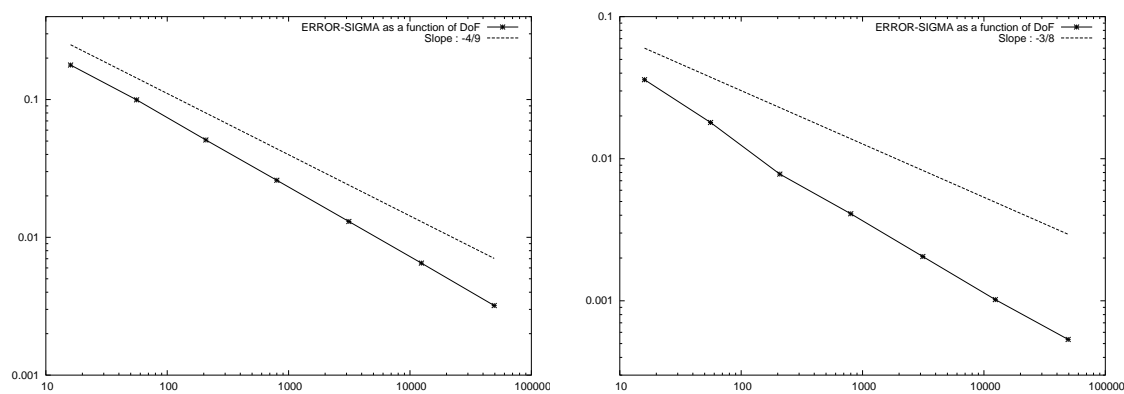

Fig. 3. $\left\|\sigma_{h}-\sigma\right\|_{0, q, \Omega}$ as a function of $D o F: p=1.8$ (left) and $p=3.0$ (right). The slope of the dashed line corresponds to predicted results and the squared-composed line to computed results.
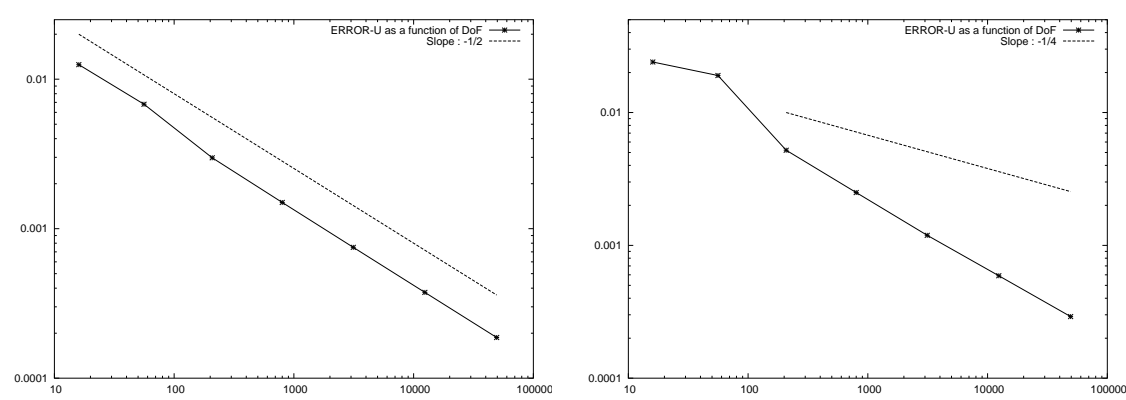

Fig. 4. $\left\|u-u_{h}\right\|_{0, p, \Omega}$ as a function of $D o F: p=1.8$ (left) and $p=3.0$ (right). The slope of the dashed line corresponds to predicted results and the squared-composed line to computed results.
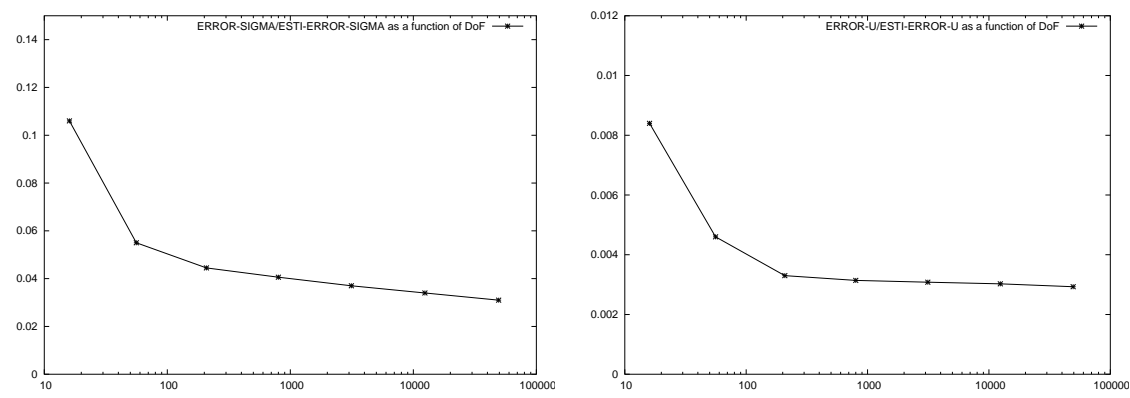

Fig. 5. $r_{1}$ (left) and $r_{2}$ (right) as a function of $D o F$ for $p=1.8$. 

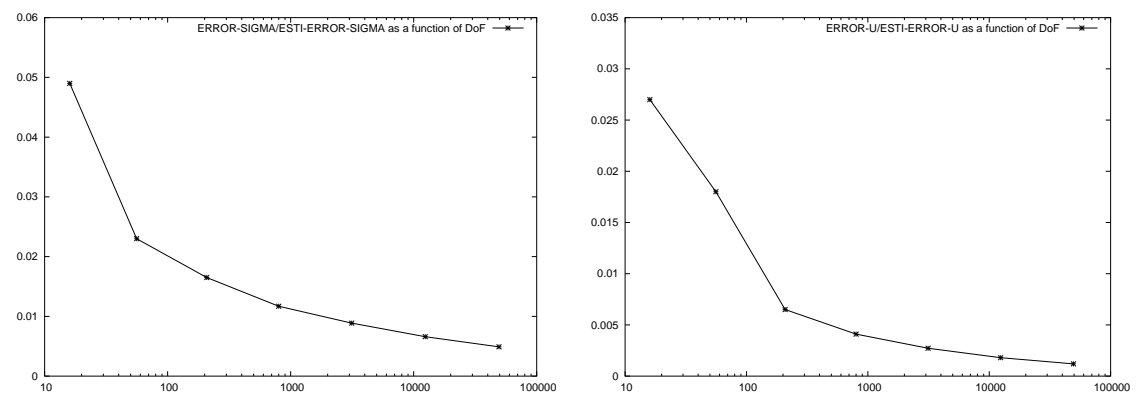

Fig. 6. $r_{3}$ (left) and $r_{4}$ (right) as a function of $D o F$ for $p=3.0$. 